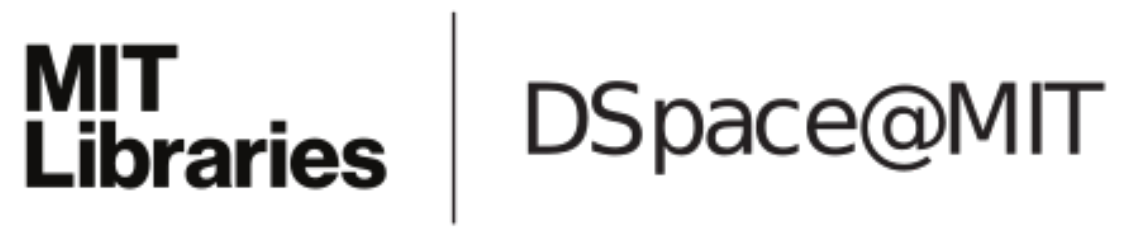

\author{
MIT Open Access Articles
}

The TWA 3 Young Triple System: Orbits, Disks, Evolution

The MIT Faculty has made this article openly available. Please share how this access benefits you. Your story matters.

Citation: Kellogg, Kendra, et al. "The TWA 3 Young Triple System: Orbits, Disks, Evolution." The Astrophysical Journal, vol. 844, no. 2, Aug. 2017, p. 168. @ 2017 The American Astronomical Society.

As Published: http://dx.doi.org/10.3847/1538-4357/aa7c60

Publisher: IOP Publishing

Persistent URL: http://hdl.handle.net/1721.1/112307

Version: Final published version: final published article, as it appeared in a journal, conference proceedings, or other formally published context

Terms of Use: Article is made available in accordance with the publisher's policy and may be subject to US copyright law. Please refer to the publisher's site for terms of use. 


\title{
The TWA 3 Young Triple System: Orbits, Disks, Evolution
}

Kendra Kellogg ${ }^{1}$, L. Prato ${ }^{2}$, Guillermo Torres ${ }^{3}$, G. H. Schaefer ${ }^{4}$, I. Avilez ${ }^{2}$, D. Ruíz-Rodríguez ${ }^{5}$, L. H. Wasserman ${ }^{2}$, Alceste Z. Bonanos ${ }^{6}$, E. W. Guenther ${ }^{7}$, R. Neuhäuser ${ }^{8}$, S. E. Levine ${ }^{2,9}$, A. S. Bosh ${ }^{2,9}$, Katie M. Morzinski ${ }^{10}$, Laird Close $^{10}$, Vanessa Bailey ${ }^{11}$, Phil Hinz ${ }^{10}$, and Jared R. Males ${ }^{10}$

${ }^{1}$ Department of Physics and Astronomy, The University of Western Ontario, London, ON N6A 3K7, Canada; kkellogg@uwo.ca

${ }^{2}$ Lowell Observatory, 1400 West Mars Hill Road, Flagstaff, AZ 86001, USA; lprato@lowell.edu

${ }^{3}$ Harvard-Smithsonian Center for Astrophysics, Cambridge, MA 02138, USA

${ }^{4}$ The CHARA Array of Georgia State University, Mount Wilson Observatory, Mount Wilson, CA 91023, USA

${ }^{5}$ Research School of Astronomy and Astrophysics, Australian National University, Canberra, ACT 2611, Australia

${ }^{6}$ IAASARS, National Observatory of Athens, 15236 Penteli, Greece

7 Thüringer Landessternwarte Tautenburg, D-07778 Tautenburg, Germany

${ }^{8}$ Astrophysikalisches Institut und Universitäts-Sternwarte, FSU Jena, Schillergäßchen 2-3, D-07745 Jena, Germany

${ }_{9}$ MIT Department of Earth, Atmospheric, and Planetary Sciences, Cambridge, MA 02139, USA

${ }^{10}$ Steward Observatory, University of Arizona, 933 N. Cherry Ave, Tucson, AZ 85721, USA

${ }^{11}$ Kavli Institute for Particle Astrophysics and Cosmology, Department of Physics, Stanford University, Stanford, CA, 94305, USA Received 2017 May 21; revised 2017 June 24; accepted 2017 June 26; published 2017 August 3

\begin{abstract}
We have characterized the spectroscopic orbit of the TWA 3A binary and provide preliminary families of probable solutions for the TWA 3A visual orbit, as well as for the wide TWA 3A-B orbit. TWA 3 is a hierarchical triple located at $34 \mathrm{pc}$ in the $\sim 10$ Myr old TW Hya association. The wide component separation is 1 ". 55 ; the close pair was first identified as a possible binary almost 20 years ago. We initially identified the 35-day period orbital solution using high-resolution infrared spectroscopy that angularly resolved the A and B components. We then refined the preliminary orbit by combining the infrared data with a reanalysis of our high-resolution optical spectroscopy. The orbital period from the combined spectroscopic solution is $\sim 35$ days, the eccentricity is $\sim 0.63$, and the mass ratio is $\sim 0.84$; although this high mass ratio would suggest that optical spectroscopy alone should be sufficient to identify the orbital solution, the presence of the tertiary B component likely introduced confusion in the blended optical spectra. Using millimeter imaging from the literature, we also estimate the inclinations of the stellar orbital planes with respect to the TWA 3A circumbinary disk inclination and find that all three planes are likely misaligned by at least $\sim 30^{\circ}$. The TWA 3A spectroscopic binary components have spectral types of M4.0 and M4.5; TWA 3B is an M3. We speculate that the system formed as a triple, is bound, and that its properties were shaped by dynamical interactions between the inclined orbits and disk.
\end{abstract}

Key words: binaries: spectroscopic - binaries: visual - circumstellar matter - stars: individual (TWA 3)

\section{Introduction}

A key question in astronomy involves the mechanisms and characteristic ages for the formation of planetary systems like our own. One approach to address this is the study of the evolution and dynamics of protoplanetary disks - the sites of planet formation around young stars. Although our own solar system is centered around a single star, it is nevertheless important to understand planet formation in multiple-star systems, not only because most stars form in binary or higher-order multiples that therefore dominate the star formation process (e.g., Duchêne \& Kraus 2013), but also because a number of exoplanetary systems have been identified in binarystar systems (e.g., Doyle et al. 2011; Kostov et al. 2014; Welsh et al. 2015). Furthermore, young multiple systems provide a means to examine how tidal interactions affect circumstellar disks. The TW Hydrae association provides an advantageous observing ground for young circumstellar disk evolution because at a distance of only $\sim 50 \mathrm{pc}$ or less, it is the nearest known group of young stars. Serendipitously, for a small stellar population (e.g., Torres et al. 2003) it contains a disproportionately large fraction of young multiples, some with complex circumstellar and circumbinary disk configurations (Webb et al. 1999; Koerner et al. 2000; Prato et al. 2001; Konopacky et al. 2007; Andrews et al. 2010).
Previous work has shown that whether a disk is circumstellar or circumbinary, the protoplanetary material usually dissipates within $\sim 10 \mathrm{Myr}$ and rarely is found to be accreting beyond that age (e.g., Haisch et al. 2001; Hernández et al. 2008). TWA 3, however, is one of the systems in the 10 Myr old TW Hydrae association that is an exception (Muzerolle et al. 2000). TWA 3 is a visual binary with a 2015 position angle of $207^{\circ}$ and a projected separation of 1".55 (Tokovinin et al. 2015), corresponding to $\sim 50$ au at a distance of $34 \pm 4 \mathrm{pc}$ (Mamajek 2005). Although different distance estimates are available in the literature, we have chosen to adopt Mamajek's estimate given the consistency with Gagné et al. (2017). The TW Hya association members demonstrate a wide range of distances, from $\sim 30$ to $50 \mathrm{pc}$, consistent with the elongated shape of the region as discussed in Kellogg et al. (2016). Basic properties of the system are listed in Table 1. Jayawardhana et al. (1999) angularly resolved the visual pair in the near- and mid-infrared (IR), demonstrating that TWA 3A exhibits optically thick excess long-wavelength emission, indicative of a dusty disk. TWA 3B appears to be devoid of dust. Furthermore, TWA 3A also has a significant gas accretion signature similar to TW Hya and TWA 14 (Muzerolle et al. 2000). Webb et al. (1999) measured $\mathrm{H} \alpha$ emission equivalent widths of 21.8 and $7.14 \AA$ for TWA 3A and 3B, respectively. Herczeg et al. (2009) found equivalent widths of 37 and $3.4 \AA$ 
Table 1

TWA 3 System Properties

\begin{tabular}{ll}
\hline \hline R.A. (J2000.0) & $11^{\mathrm{h}} 10^{\mathrm{m}} 27^{\mathrm{s}} .88$ \\
Decl. (J2000.0) & $-37^{\mathrm{d}} 31^{\mathrm{m}} 52^{\mathrm{s}} 0$ \\
Distance (pc) & $34 \pm 4^{\mathrm{a}}$ \\
A-B separation (2015) & $15^{\prime \prime} 55^{\mathrm{b}}$ \\
A-B position angle (2015) & $207^{\mathrm{ob}}$ \\
$V$ (mag) & $12.04 \pm 0.01^{\mathrm{c}}$ \\
$J$ (mag) & $7.651 \pm 0.019^{\mathrm{d}}$ \\
$H$ (mag) & $7.041 \pm 0.027^{\mathrm{d}}$ \\
$K_{s}$ (mag) & $6.774 \pm 0.020^{\mathrm{d}}$ \\
$\mathrm{A}_{V}$ & $0.01^{\mathrm{e}}$ \\
\hline
\end{tabular}

Notes.

a Mamajek (2005).

b Tokovinin et al. (2015).

c Torres et al. (2000)

d 2MASS.

e McJunkin et al. (2014).

for TWA $3 \mathrm{~A}$ and $3 \mathrm{~B}$, respectively. Given the 10 and $20 \AA$ approximate limits for early and late $\mathrm{M}$ stars with active disks (Martín 1998), the B component is likely not accreting, while the A component is a bona fide, classical T Tauri star (CTTS). However, Muzerolle et al. (2000) calculated a relatively low gas accretion rate of $\sim 5 \times 10^{-11} M_{\odot} \mathrm{yr}^{-1}$ for TWA 3A. Although Herczeg et al. (2009) used the Balmer continuum emission from TWA 3A to measure a larger accretion rate of $2.5 \times 10^{-10} M_{\odot} \mathrm{yr}^{-1}$, typical accretion rates for CTTSs range from $10^{-9}$ to $10^{-7} M_{\odot} \mathrm{yr}^{-1}$ (Bouvier et al. 2007). This modest accretion rate and the relatively low intensity X-ray emission (Huenemoerder et al. 2007), coupled with indications for a dust gap in the inner disk (Jayawardhana et al. 1999; Andrews et al. 2010), suggest that the disk around the A component is likely a pre-transition disk, about to evolve from a CTTS to a weaklined $\mathrm{T}$ Tauri star (WTTS) or to a diskless system like TWA 3B.

Measurements at $10 \mu \mathrm{m}$ have shown the presence of silicates in the TWA 3A disk, suggesting some grain growth (Uchida et al. 2004). Andrews et al. (2010) resolved the disk in continuum measurements at $880 \mu \mathrm{m}$. Their data show no evidence for warm dust around the B component or surrounding the entire system. Combining their data with a broadband spectral energy distribution (SED) and comparing to models of truncated disks, Andrews et al. estimated an outer disk radius of $\sim 15-25$ au and an inner radius of $\sim 1$ au, consistent with the 1.3 au estimate of Uchida et al. (2004).

Webb et al. (1999) suggested, and Muzerolle et al. (2000) and Torres et al. (2003) confirmed, that TWA 3A is a spectroscopic binary, but one for which an orbital solution has been elusive. Substantial optical data of the unresolved system, described in Torres et al., were collected at several facilities on this system over 2 decades by several of us. Following the speculation that $\mathrm{A}$ is itself a spectroscopic binary in Webb et al., beginning in 2002 one of us also obtained occasional observations of the system with the NIRSPEC high-resolution IR spectrograph at the Keck II telescope. This approach provided important advantages: the superior seeing typical on Maunakea, in conjunction with a $10 \mathrm{~m}$ telescope, allowed for straightforward angular resolution of the visual binary pair on a regular basis. Furthermore, because luminosity is a steeper function of mass in the optical compared to the IR, longerwavelength observations facilitate identification of the
Table 2

Facilities and Observations

\begin{tabular}{ll}
\hline \hline 1.5 m Tillinghast + echelle (CfA) & $R=35,000$ optical spectroscopy \\
4.5 m MMT + echelle (CfA) & $R=35,000$ optical spectroscopy \\
1.5 m ESO + FEROS & $R=44,000$ optical spectroscopy \\
2.5 m du Pont + echelle & $R=40,000$ optical spectroscopy \\
10 m Keck II + NIRSPEC & $R=30,000$ IR spectroscopy \\
10 m Keck II + NIRSPAO & $R=30,000$ IR spectroscopy with AO \\
4.3 m DCT + LMI & $B V R I$ imaging \\
6.0 m Magellan + Clio & HK AO imaging \\
\hline
\end{tabular}

spectrum of a fainter and redder secondary star in the blended lines of the TWA 3A spectroscopic binary (Prato et al. 2002a).

This approach has yielded an orbital solution for the TWA $3 \mathrm{~A}$ system and a mass ratio measurement for the pair, increasing the small pool of double-lined solutions for premain-sequence spectroscopic binaries (e.g., Rosero et al. 2011). With the advent of ALMA and GAIA, increasingly sensitive techniques will provide the potential means for measuring the total system mass using the velocity curve of a circumbinary disk (Guilloteau et al. 2014) or using the astrometric motion of a binary photocenter to measure the orbital inclination (e.g., Goldin \& Makarov 2007). Angularly resolved visual orbits from adaptive optics (AO) imaging and interferometry also play an important role (e.g., Simon et al. 2013; Le Bouquin et al. 2014). In conjunction with the mass ratio, these advances will allow for the determination of the individual component masses, crucial input to models of young star evolution (Prato et al. 2002b).

The confirmation of the TWA 3A spectroscopic binary demonstrates that its long-lived disk is circumbinary. A standard paradigm for disk evolution in young hierarchical triple systems anticipates star-disk tidal interactions that disrupt circumbinary material, while circumstellar material surrounding the single star remains intact and continues to accrete onto the central star for a longer period of time (White et al. 2002). Although one might expect circumbinary disk disruption to be a function of stellar separation, only a handful of young spectroscopic binaries have circumbinary material, and the orbital periods of these pairs range from a couple of days to almost a year. TWA 3 is an intriguing example of a system with both a single and a binary and a long-lived disk only around the binary; this triple provides potentially valuable clues to understanding important but subtle aspects of disk evolution around low-mass stars. In Section 2 we describe the visible light and IR observations and data reduction, and in Section 3 we provide the details of the radial velocity (RV) analysis and results. We provide a description in Section 4 of the hierarchical visual and spectroscopic orbits in the system. A description of the structure and origin of this complex system appears in Section 5; these results are discussed in Section 6, and Section 7 provides a summary.

\section{Observations}

We report on close to 2 decades of imaging and spectroscopy of the TWA 3 system. Table 2 lists the facilities and instrumentation that supplied the data sets described below. 
Table 3

Keck Infrared Spectroscopy

\begin{tabular}{|c|c|c|}
\hline $\begin{array}{l}\text { UT } \\
\text { Date }\end{array}$ & $\begin{array}{l}\text { Component } \\
\text { Observed }\end{array}$ & $\mathrm{AO}$ ? \\
\hline 2002 Dec 22 & $\mathrm{~A}, \mathrm{~B}$ & \\
\hline 2003 Feb 08 & A, B & \\
\hline 2003 Apr 20 & A & yes \\
\hline 2004 Jan 26 & A & \\
\hline 2004 Dec 26 & A & \\
\hline 2005 Feb 22 & A & \\
\hline 2009 Dec 06 & A, B & yes \\
\hline 2010 Feb 24 & A & \\
\hline 2010 Dec 12 & $\mathrm{~A}, \mathrm{~B}$ & yes \\
\hline 2011 Feb 05 & A & \\
\hline 2011 Feb 09 & A & \\
\hline
\end{tabular}

\subsection{Near-Infrared}

\subsubsection{Spectroscopy}

Near-IR, spectroscopic observations were made over 11 epochs between 2002 December and 2011 February with the NIRSPEC instrument on the Keck II $10 \mathrm{~m}$ telescope, located on Maunakea. The UT dates of observation are listed in Table 3. NIRSPEC is a near-IR, cross-dispersed, cryogenic spectrograph and employs a $1024 \times 1024$ ALADDIN InSb array detector. Our data were taken in the $H$ band with a central wavelength of $\sim 1.555 \mu \mathrm{m}$. We used the 0 ". 288 slit, yielding a resolution of $R \approx 30,000$. TWA 3 has a Two Micron All Sky Survey (2MASS) $H$-band magnitude of 7.041 (Table 1). Integration times varied between 20 and $180 \mathrm{~s}$, depending on seeing conditions and whether or not NIRSPEC was employed behind the AO system, which reduces the total throughput by a factor of $\sim 5$ because of the addition of numerous reflecting surfaces on the AO bench. We nodded the telescope between two positions on the slit in an $\mathrm{AB}$ or an $\mathrm{ABBA}$ nod sequence to allow for background subtraction between sequential spectra.

All near-IR spectroscopic data reductions were made with the REDSPEC package ${ }^{12}$ (Kim et al. 2015). We analyzed an order centered around $1.555 \mu \mathrm{m}$ in the $H$ band, a convenient region because it is devoid of all atmospheric absorption lines, therefore eliminating the need to divide by a telluric standard star in the reduction process. There are also strong $\mathrm{OH}$ nightsky emission lines spaced at relatively regular intervals across this order (Rousselot et al. 2000), useful for determining the spectral dispersion solution. The procedure for using REDSPEC is described in detail by Prato et al. (2002a). In brief, a median-filtered cube of dark frames was subtracted from a median-filtered cube of flat frames to create a master flat, which was then divided into A-B subtracted pairs. Using fits to the spectral traces and the night-sky $\mathrm{OH}$ emission lines or comparison lamp lines, the software rectified this semiprocessed frame and determined the wavelength zero-point and dispersion solution. Spectra were then extracted by summing rows at the location of the stellar signal, the number of which depends on the seeing at the time of observation. The two components of TWA 3, when observed simultaneously along the slit in 2002 December, 2003 February, 2009 December, and 2010 December, were well separated. Once extracted, the spectra were cleaned of bad pixels, flattened, normalized, and corrected for barycentric motion. Figures 1 and 2 show the final

\footnotetext{
${ }^{12}$ See https://www2.keck.hawaii.edu/inst/nirspec/redspec.html.
}

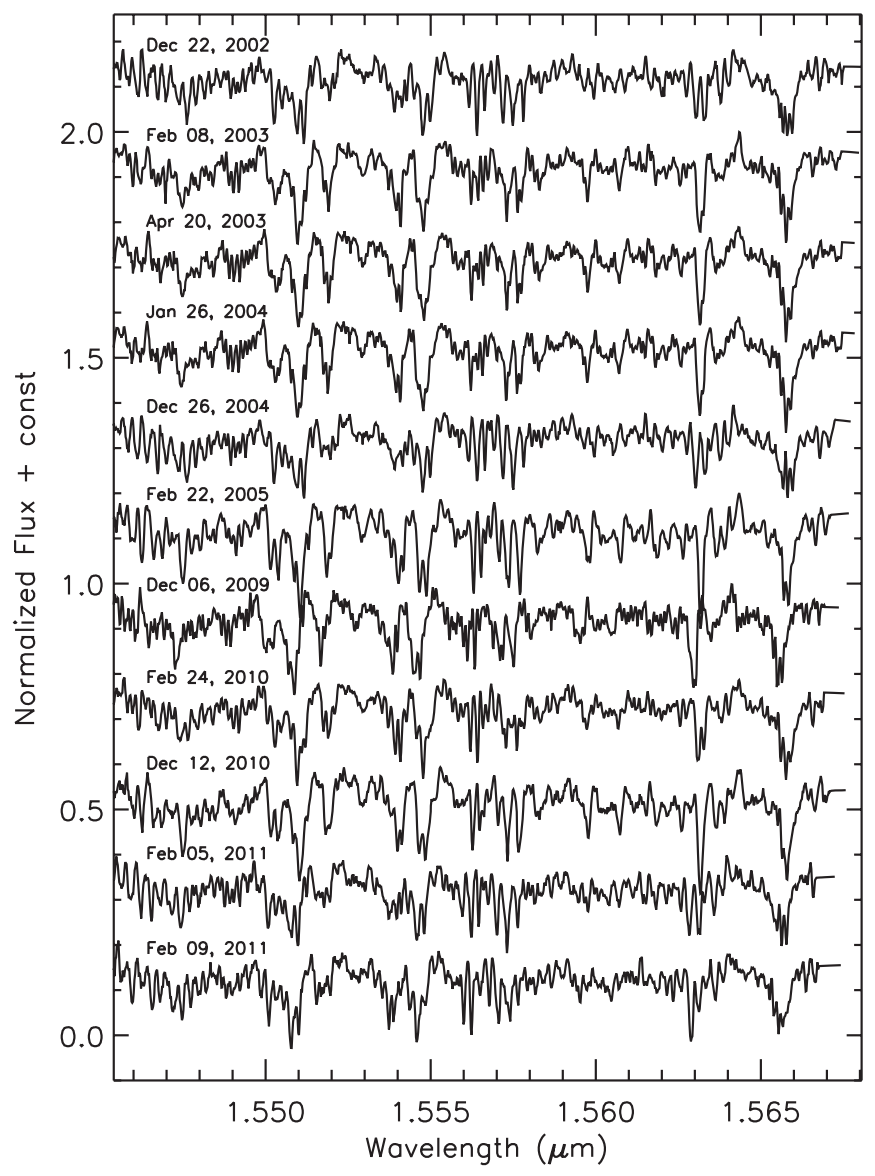

Figure 1. Near-IR spectra of TWA 3A from all 11 epochs. The spectra have been normalized to unity, barycentric corrected, and displaced along the vertical axis for viewing purposes by the addition of a constant. The UT dates of observation are given above each spectrum. Effectively every detectable feature is real; the signal-to-noise ratio ranges from $\sim 150$ to several hundred. The doubling of the lines is evident in at least half of the epochs.

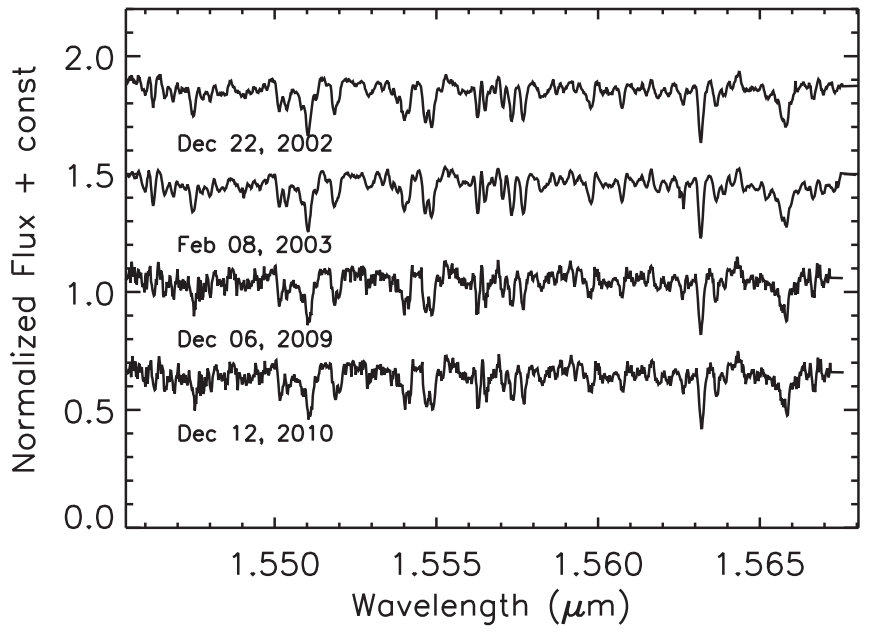

Figure 2. Spectra of TWA 3B from all epochs, plotted as the $3 \mathrm{~A}$ spectra in Figure 1. The signal-to-noise ratio in the 2009 and 2010 data is $~ 100$; yet again all the discernible features are likely real.

spectra from all our observations of TWA $3 \mathrm{~A}$ and $3 \mathrm{~B}$, respectively. Effectively every feature in these spectra is real; the signal-to-noise ratio was typically $>200$. The double-lined nature of the TWA $3 \mathrm{~A}$ spectra is prominent at most epochs. In the following we refer to the two components as TWA 3Aa and 
Table 4

Position Measurements of TWA 3B relative to TWA 3A

\begin{tabular}{|c|c|c|c|c|c|c|}
\hline UT Date & $\mathrm{BY}^{\mathrm{a}}$ & $\rho$ (mas) & P.A. (deg) & Telescope & Filter & Flux Ratio \\
\hline 2014 Apr 21 02:10 & 2014.3030 & $1.5440 \pm 0.0048$ & $207.65 \pm 0.18$ & Magellan & $\begin{array}{l}H \\
K_{s}\end{array}$ & $\begin{array}{l}0.689 \pm 0.017 \\
0.650 \pm 0.034\end{array}$ \\
\hline 2014 Apr 08 05:45 & 2014.2678 & $1.5124 \pm 0.0181$ & $207.387 \pm 0.687$ & DCT & $\begin{array}{l}B \\
V \\
R \\
I\end{array}$ & $\begin{array}{l}0.8185 \pm 0.0070 \\
0.7543 \pm 0.0030 \\
0.7216 \pm 0.0023 \\
0.6987 \pm 0.0049\end{array}$ \\
\hline
\end{tabular}

Note.

${ }^{\mathrm{a}}$ Besselian Year.

TWA $3 \mathrm{Ab}$. No line doubling is detectable in the spectra of TWA 3B.

\subsubsection{Imaging}

We observed TWA 3 in the $H$ and $K_{S}$ bands with the Clio imager at the Magellan $6.5 \mathrm{~m}$ telescope using AO on UT 2014 April 21. Integration times were $0.1 \mathrm{~s}$ at $H$ and $0.3 \mathrm{~s}$ at $K_{S}$. The images were flat-fielded, and pairs of dithered images were subtracted to remove the sky background and bad pixels. As the TWA $3 \mathrm{~A}$ and $\mathrm{B}$ components were well separated in the AO images, we used the unresolved TWA 3A point-spread function (PSF) to measure the relative position and flux ratio of TWA 3B. We used a plate scale of $15.846 \pm 0.043$ mas pixel $^{-1}$ and a rotation angle offset of $-1.797 \pm 0.159$ (Morzinski et al. 2015). Table 4 lists the separation, position angle, and flux ratios measured from these observations. No evidence was seen indicating that the $\mathrm{A}$ and $\mathrm{B}$ components are themselves visual binaries.

\subsection{Optical}

\subsubsection{Spectroscopy}

TWA 3 was placed on the observing program at the HarvardSmithsonian Center for Astrophysics (CfA) in 1998, along with other members of the TW Hya association. It was monitored between January of that year and 2007 February with two nearly identical echelle spectrographs (Digital Speedometers; Latham 1992) attached to the $1.5 \mathrm{~m}$ Tillinghast reflector at the F. L. Whipple Observatory (Mount Hopkins, AZ) and to the $4.5 \mathrm{~m}$ equivalent Multiple Mirror Telescope (also on Mount Hopkins), prior to its conversion to a $6.5 \mathrm{~m}$ monolithic mirror. A single echelle order $45 \AA$ wide was recorded with intensified Reticon photon-counting detectors, at a central wavelength of about $5190 \AA$. This order contains the lines of the Mg I b triplet. A total of 35 usable exposures of the star were obtained at a resolving power of $R \approx 35,000$, with integration times ranging from 600 to $2400 \mathrm{~s}$. Given the northern location of these telescopes, all observations were made at airmasses larger than about 2.8 and are rather weak: the signal-to-noise ratios range from 7 to 15 per resolution element of $8.5 \mathrm{~km} \mathrm{~s}^{-1}$. Nevertheless, as we show later, this is sufficient to derive meaningful RVs. The spectrograph slit was $1^{\prime \prime}$, but the seeing at low elevation was often very poor. Consequently, despite the angular separation of 1 ". 55 between TWA 3A and TWA 3B, all of these spectra are likely to include light from both visual components under typical seeing conditions. Thorium-argon spectra were obtained before and after each science exposure to set the wavelength scale. The zero-point of the CfA velocity system was monitored by taking exposures of the dusk and dawn sky, and small run-to-run corrections for instrumental shifts were applied to the velocities described later, following Latham (1992).

Additional optical spectra were collected with the fiber-fed FEROS spectrograph mounted on the ESO $1.5 \mathrm{~m}$ telescope (La Silla, Chile). These observations cover the approximate wavelength range from 3600 to $9200 \AA$ at a resolving power of 44,000. Integration times ranged from 600 to $1800 \mathrm{~s}$. We obtained a total of eight usable exposures between 1999 March and 2000 July, with signal-to-noise ratios of 25-35 per pixel around $5200 \AA$. Standard calibration frames (Th-Ar-Ne lamp, flat-field, and bias exposures) were taken at the beginning of each night, and the data reduction was performed under MIDAS using the FEROS pipeline. Telluric lines were used to compensate for instrumental shifts, and additionally observations were made each night of the standard star HR 5777 (Murdoch et al. 1993) to check for RV shifts. These were found to be always smaller than about $30 \mathrm{~m} \mathrm{~s}^{-1}$. Because the spectrograph fiber has a $2^{\prime \prime}$ diameter, all FEROS spectra also likely contain light of both visual components of TWA 3 .

Further observations of the star were obtained with the echelle spectrograph on the $2.5 \mathrm{~m}$ du Pont telescope at the Las Campanas Observatory (Chile). Nine observations were gathered in 2006 from UT January 30 to February 5. The wavelength coverage is $3500-10000 \AA$ in 64 orders, and with a slit width of $1^{\prime \prime}$ the resolving power is approximately 40,000. Exposure times were $900 \mathrm{~s}$, and the signal-to-noise ratios averaged $35-40$ per pixel in order 32 centered around $5170 \AA$. Thorium-argon lamp exposures were taken before or after each science exposure, and standard reductions were performed with IRAF (bias subtraction, flat-fielding). As with the CfA and FEROS spectra, under typical seeing conditions most of the exposures probably include light from both visual components of TWA 3, even though the slit used was $1^{\prime \prime}$ wide. On the first night, however, the seeing was good enough to permit separate spectra of each star with little contamination from the other.

\subsubsection{Imaging}

Optical imaging was carried out at Lowell Observatory's $4.3 \mathrm{~m}$ Discovery Channel Telescope (DCT) with the Large Monolithic Imager on UT 2014 April 8 using Johnson $B$ and $V$ and Cousins $R$ and $I$ filters; target exposure times were 30, 15, 5 , and $1.25 \mathrm{~s}$, respectively. Three $2 \times 2$ binned images were taken of TWA 3 in each band. Mean bias and flat frames were created and applied to the individual target exposures. We cross-registered images in each individual band to align them and combined each set of three images, trimming the final reduced output in each band to $3000 \times 3000$ pixels for analysis. 
Table 5

Template Spectral Type Standard Stars

\begin{tabular}{llccc}
\hline \hline Object & $\begin{array}{l}\text { Spectral } \\
\text { Type }\end{array}$ & $\begin{array}{c}\mathrm{RV} \\
\left(\mathrm{km} \mathrm{s}^{-1}\right)\end{array}$ & $\begin{array}{c}v \sin i \\
\left(\mathrm{~km} \mathrm{~s}^{-1}\right)\end{array}$ & $\begin{array}{c}T_{\text {eff }}{ }^{\mathrm{a}} \\
\mathrm{K}\end{array}$ \\
\hline Infrared & & & \\
\hline GJ 15A & M3 & 9.6 & $<2.5^{\mathrm{c}}$ & $3453 \pm 86$ \\
GJ 402 & M4 & -3.1 & $<2.5^{\mathrm{c}}$ & $3238 \pm 60$ \\
GJ 669B & M4.5 & -36.8 & $6.1^{\mathrm{c}}$ & $3131 \pm 85$ \\
\hline Optical & & & & \\
\hline GJ 48 & M3.0V & 2.49 & $<2.5^{\mathrm{d}}$ & $3453 \pm 86$ \\
GJ 699 & M4.0V & -108.77 & $<2.5^{\mathrm{d}}$ & $3238 \pm 60$ \\
\hline
\end{tabular}

Notes.

${ }^{a}$ From or derived from Mann et al. (2015).

b Prato (2007).

c Reiners et al. (2012).

d Browning et al. (2010).

Average seeing of $\sim 0 . \prime 9$ facilitated the extraction of photometry for the individual TWA $3 \mathrm{~A}$ and $3 \mathrm{~B}$ components. Although without AO the individual PSFs were overlapping, we applied the PSF of a nearby single star to model the relative positions and flux ratio of the pair using techniques described in Schaefer et al. (2014). The binned plate scale ${ }^{13}$ was 0 ". 24 pixel $^{-1}$. Results of these observations are given in Table 4.

\section{Radial Velocities}

\subsection{Near-IR}

To measure RVs of TWA $3 \mathrm{Aa}$ and $3 \mathrm{Ab}$, we used a two-dimensional cross-correlation code written at Lowell Observatory following the TODCOR algorithm originally developed by Zucker \& Mazeh (1994). This algorithm determines the individual RVs of both stars in the spectroscopic binary simultaneously. It requires two templates, one for each component, for which either synthetic or observed spectra may be used. For this work we used observed spectra of mainsequence stars of spectral types similar to those of our $\mathrm{T}$ Tauri stars (Prato et al. 2002a; Bender et al. 2005). A nonlinear, limbdarkened broadening kernel (e.g., Bender \& Simon 2008) was applied to each template to mimic spectra with different rotational velocities. The spectral types, RVs, $v \sin i$, and $T_{\text {eff }}$ values of the template stars we used to optimize the crosscorrelation with the TWA 3A IR spectra, GJ 402 and GJ 669B, appear in Table 5. Templates broadened to $v \sin i$ values of $0-15 \mathrm{~km} \mathrm{~s}^{-1}$ were tested; we found best fits using the M4 template GJ 402, broadened to a $v \sin i$ of $7 \mathrm{~km} \mathrm{~s}^{-1}$, and the M4.5 template GJ 669B, broadened to $5 \mathrm{~km} \mathrm{~s}^{-1}$, for the spectra of TWA $3 \mathrm{Aa}$ and $\mathrm{Ab}$, respectively. However, these values fall below our 2-pixel velocity resolution element of $\sim 8 \mathrm{~km} \mathrm{~s}^{-1}$, and thus we are limited to a general statement that the TWA $3 \mathrm{Aa}$ and $\mathrm{Ab}$ components have $v \sin i$ values less than $8 \mathrm{~km} \mathrm{~s}^{-1}$ (but see Section 3.2). For TWA 3B, we found a maximum in the cross-correlation coefficient for a $v \sin i$ value of $15 \mathrm{~km} \mathrm{~s}^{-1}$. Given the high signal-to-noise spectra obtained in the IR and the low internal uncertainty in the RV measurements of $0.2 \mathrm{~km} \mathrm{~s}^{-1}$, we estimate that the largest uncertainty introduced into our RV measurements of the TWA

\footnotetext{
13 http://www2.lowell.edu/rsch/LMI/specs.html
}

Table 6

Keck IR Radial Velocity Measurements of TWA 3

\begin{tabular}{lcrrr}
\hline \hline $\begin{array}{l}\mathrm{HJD} \\
(2,400,000+)\end{array}$ & $\begin{array}{c}\text { Orbital } \\
\text { Phase }\end{array}$ & $\begin{array}{c}\mathrm{RV}_{\mathrm{Aa}} \\
\left(\mathrm{km} \mathrm{s}^{-1}\right)\end{array}$ & $\begin{array}{c}\mathrm{RV}_{\mathrm{Ab}} \\
\left(\mathrm{km} \mathrm{s}^{-1}\right)\end{array}$ & $\begin{array}{c}\mathrm{RV}_{\mathrm{B}} \\
\left(\mathrm{km} \mathrm{s}^{-1}\right)\end{array}$ \\
\hline $52,631.1403$ & 0.895 & 31.22 & -16.84 & 7.14 \\
$52,679.0055$ & 0.267 & -3.66 & 21.03 & 7.09 \\
$52,749.7738$ & 0.296 & 0.00 & 22.07 & $\ldots$ \\
$53,031.0457$ & 0.361 & 1.53 & 17.70 & $\ldots$ \\
$53,366.1060$ & 0.967 & 32.25 & -20.59 & $\ldots$ \\
$53,423.9356$ & 0.625 & 12.45 & 3.01 & $\ldots$ \\
$55,172.1516$ & 0.748 & 18.68 & -4.97 & 7.64 \\
$55,251.9446$ & 0.036 & -7.34 & 27.51 & $\ldots$ \\
$55,543.1431$ & 0.385 & 1.81 & 15.55 & 7.79 \\
$55,597.9402$ & 0.956 & 34.40 & -20.58 & $\ldots$ \\
$55,601.9331$ & 0.071 & -12.17 & 33.82 & $\ldots$ \\
\hline
\end{tabular}

Note. RV uncertainties are $\sigma_{\mathrm{Aa}}=0.63 \mathrm{~km} \mathrm{~s}^{-1}, \sigma_{\mathrm{Ab}}=0.85 \mathrm{~km} \mathrm{~s}^{-1}$, and $\sigma_{\mathrm{B}}=0.59 \mathrm{~km} \mathrm{~s}^{-1}$ (see text).

3A components comes from the uncertainty in the template RVs (Mazeh et al. 2003).

The TODCOR algorithm can also solve for the intensity ratio between the components. The intensity ratio when the stars are at their largest RV separation gives the best results, when available. We first removed the two sets of points with RVs closest to the center-of-mass velocity for the system, taken on UT 2005 February 22 (JD 2,453,423.9356) and UT 2010 December 12 (JD 2,455,543.1431), and left the light ratio as a free parameter for the other nine TWA 3A spectra. We obtained an average light ratio of $0.61 \pm 0.11$. This value was then fixed and the component RVs were redetermined with TODCOR for all 11 spectra. The resulting heliocentric RVs extracted from our IR spectra of TWA 3A using M4V and M4.5V template stars (Table 5) are listed in Table 6. The RVs for TWA 3B, determined from cross-correlation against GJ 15A (Table 5), also appear in Table 6 and show an RV range of $0.70 \mathrm{~km} \mathrm{~s}^{-1}$. Cross-correlating the strongest spectrum of TWA 3B from UT 2002 December 22 (Figure 2) against those from the three other epochs indicated no RV shift greater than $0.3 \mathrm{~km} \mathrm{~s}^{-1}$ over a period of almost $8 \mathrm{yr}$. The standard deviation of the TWA $3 \mathrm{~B}$ $\Delta(\mathrm{RV})$ measurements from these cross-correlations was $0.2 \mathrm{~km} \mathrm{~s}^{-1}$, indicative of our internal $\mathrm{RV}$ uncertainties for the IR spectroscopy of these bright target stars.

\subsection{Optical}

In view of the composite nature of our CfA spectra (TWA $3 \mathrm{~A}+$ TWA 3B) and the knowledge from our near-IR observations that one of the visual components is doublelined, we analyzed the CfA spectra using TRICOR (Zucker et al. 1995), a three-dimensional extension of the twodimensional cross-correlation algorithm TODCOR (Zucker \& Mazeh 1994). TRICOR uses three (possibly different) templates, one for each star. We selected these templates from among a set of spectra of $M$ dwarfs taken with the same instrumentation as the target, covering a wide range of spectral types (K7-M5.5). Our choice was guided by the spectral types of the best templates found from the analysis of the near-IR spectra and by extensive testing to identify the closest match to the optical spectra as indicated by the highest cross-correlation values averaged over all our CfA exposures. The best compromise among the available templates yielded GJ 699 (Barnard's star) for the two components of the double-lined 
Table 7

CfA Radial Velocity Measurements of TWA 3

\begin{tabular}{|c|c|c|c|c|c|c|c|}
\hline $\begin{array}{l}\text { HJD } \\
(2,400,000+)\end{array}$ & $\begin{array}{l}\text { Orbital } \\
\text { Phase }\end{array}$ & $\begin{array}{c}\mathrm{RV}_{\mathrm{Aa}} \\
\left(\mathrm{km} \mathrm{s}^{-1}\right)\end{array}$ & $\begin{array}{c}\mathrm{RV}_{\mathrm{Ab}} \\
\left(\mathrm{km} \mathrm{s}^{-1}\right)\end{array}$ & $\begin{array}{c}\mathrm{RV}_{\mathrm{B}} \\
\left(\mathrm{km} \mathrm{s}^{-1}\right)\end{array}$ & $\begin{array}{c}\sigma_{\mathrm{Aa}} \\
\left(\mathrm{km} \mathrm{s}^{-1}\right)\end{array}$ & $\begin{array}{c}\sigma_{\mathrm{Ab}} \\
\left(\mathrm{km} \mathrm{s}^{-1}\right)\end{array}$ & $\begin{array}{c}\sigma_{\mathrm{B}} \\
\left(\mathrm{km} \mathrm{s}^{-1}\right)\end{array}$ \\
\hline $50,828.9583$ & 0.225 & -4.28 & 26.71 & 5.94 & 4.10 & 6.66 & 3.95 \\
\hline $51,177.0639$ & 0.205 & -3.93 & 23.00 & 7.29 & 2.90 & 4.71 & 2.80 \\
\hline $51,237.9189$ & 0.950 & 39.18 & -13.93 & 12.90 & 2.65 & 4.30 & 2.55 \\
\hline $51,595.9255$ & 0.215 & -4.61 & 24.70 & 10.15 & 2.65 & 4.30 & 2.55 \\
\hline $51,619.8713$ & 0.901 & 33.65 & -14.36 & 9.47 & 2.77 & 4.49 & 2.67 \\
\hline $51,621.8603$ & 0.958 & 37.45 & -25.56 & 4.56 & 2.77 & 4.49 & 2.67 \\
\hline $51,682.7086$ & 0.703 & 16.36 & 3.66 & 14.07 & 2.37 & 3.84 & 2.28 \\
\hline $51,917.0057$ & 0.420 & 0.90 & 23.18 & 10.16 & 2.77 & 4.49 & 2.67 \\
\hline $51,945.9559$ & 0.250 & -3.11 & 28.13 & 9.82 & 2.29 & 3.72 & 2.21 \\
\hline $51,947.9030$ & 0.306 & -1.91 & 26.98 & 8.36 & 4.10 & 6.66 & 3.95 \\
\hline $51,971.8490$ & 0.993 & 17.30 & 2.20 & 7.61 & 2.65 & 4.30 & 2.55 \\
\hline $51,972.8516$ & 0.021 & 0.66 & 24.23 & 10.28 & 2.77 & 4.49 & 2.67 \\
\hline $51,973.8552$ & 0.050 & -9.99 & 36.49 & 11.10 & 3.06 & 4.97 & 2.95 \\
\hline $52,008.7574$ & 0.051 & -3.75 & 36.49 & 7.67 & 2.29 & 3.72 & 2.21 \\
\hline $52,009.7560$ & 0.079 & -12.87 & 34.92 & 8.09 & 2.29 & 3.72 & 2.21 \\
\hline $52,010.7549$ & 0.108 & -10.12 & 37.06 & 6.40 & 2.77 & 4.49 & 2.67 \\
\hline $52,011.7852$ & 0.138 & -9.87 & 32.41 & 7.45 & 2.16 & 3.51 & 2.08 \\
\hline $52,034.6899$ & 0.794 & 23.48 & -6.55 & 9.00 & 2.10 & 3.42 & 2.02 \\
\hline $52,036.6749$ & 0.851 & 30.59 & -3.31 & 12.04 & 2.23 & 3.61 & 2.15 \\
\hline $52,037.6722$ & 0.880 & 32.22 & -13.52 & 9.91 & 2.37 & 3.84 & 2.28 \\
\hline $52,038.6889$ & 0.909 & 30.09 & -17.56 & 3.97 & 2.65 & 4.30 & 2.55 \\
\hline $52,039.7160$ & 0.938 & 34.12 & -23.75 & 9.06 & 3.24 & 5.27 & 3.12 \\
\hline $52,336.8646$ & 0.458 & 4.67 & 5.77 & 14.82 & 4.10 & 6.66 & 3.95 \\
\hline $52,360.7904$ & 0.144 & -4.51 & 33.18 & 8.67 & 2.55 & 4.13 & 2.46 \\
\hline $52,395.6929$ & 0.145 & -11.35 & 23.39 & 6.37 & 3.47 & 5.63 & 3.35 \\
\hline $52,653.9864$ & 0.550 & 4.61 & 5.81 & 14.23 & 3.75 & 6.08 & 3.62 \\
\hline $52,722.7959$ & 0.523 & 8.55 & 10.74 & 11.38 & 2.90 & 4.71 & 2.80 \\
\hline $52,771.6831$ & 0.925 & 28.34 & -20.69 & 5.19 & 3.24 & 5.27 & 3.12 \\
\hline $53,016.9925$ & 0.958 & 36.04 & -25.21 & 8.23 & 1.87 & 3.04 & 1.80 \\
\hline $53,035.9465$ & 0.501 & 9.31 & 14.46 & 4.42 & 2.45 & 3.98 & 2.36 \\
\hline $53,127.7003$ & 0.132 & -5.98 & 34.88 & 9.67 & 2.16 & 3.51 & 2.08 \\
\hline $53,479.7427$ & 0.225 & -4.89 & 15.67 & 6.14 & 2.90 & 4.71 & 2.80 \\
\hline $53,781.9036$ & 0.889 & 35.70 & -16.91 & 10.66 & 2.37 & 3.84 & 2.28 \\
\hline $53,864.6736$ & 0.262 & -1.47 & 20.14 & 7.03 & 2.55 & 4.13 & 2.46 \\
\hline $54,136.9247$ & 0.067 & -10.99 & 31.18 & 9.40 & 2.77 & 4.49 & 2.67 \\
\hline
\end{tabular}

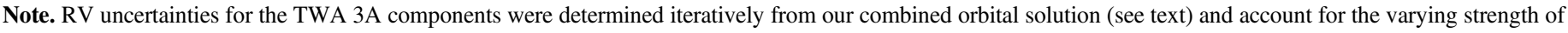
each spectrum.

binary TWA 3A and GJ 48 for TWA 3B, in good agreement with the spectral type standards used for the IR analysis (Table 5). Changing these templates by one subtype resulted in relatively minor differences in the velocities and did not change our final solution significantly. We added rotational broadening to the templates with $v \sin i$ values of 7,5 , and $12 \mathrm{~km} \mathrm{~s}^{-1}$ for TWA 3Aa, Ab, and B, respectively, informed by those used for the Keck spectra.

Thirteen of our CfA observations were contaminated by moonlight. In order to prevent biases in the velocities, these spectra were analyzed with an extension of TRICOR to four dimensions (QUADCOR; Torres et al. 2007), selecting as the fourth template a synthetic spectrum corresponding to the Sun, based on model atmospheres by R. L. Kurucz. We checked that in each case the velocity for this fourth set of lines agreed with that expected from the barycentric motion of Earth. The measured CfA velocities for the three components of TWA 3 transformed to the heliocentric frame are reported in Table 7. Typical uncertainties are about 2.7, 4.4, and $2.7 \mathrm{~km} \mathrm{~s}^{-1}$ for TWA $3 \mathrm{Aa}, \mathrm{Ab}$, and $\mathrm{B}$, respectively. Because of significant differences in the line strengths in each spectrum, the individual uncertainties take into account the signal-to-noise ratio of each observation. Following Torres et al. (2007), we determined the light ratio between $\mathrm{Ab}$ and $\mathrm{Aa}$ to be $0.59 \pm 0.08$ at the mean wavelength of our observations (5190 $\mathrm{A})$. While in principle the TRICOR/QUADCOR analysis can also provide the light ratio between stars $\mathrm{B}$ and $\mathrm{Aa}$, in practice this measurement is unreliable because of slit losses. ${ }^{14}$ The $\mathrm{Ab} / \mathrm{Aa}$ light ratio is unaffected because the angular separation between those stars is negligible compared to the slit width.

Similar analysis techniques were applied to the FEROS and $\mathrm{du}$ Pont spectra, using the same templates as above in view of the similar resolving power of the instruments. For the du Pont spectrum in which TWA 3A was observed separately from TWA 3B, we applied TODCOR, as the spectrum is only double-lined; for TWA 3B alone, the RV was derived by onedimensional cross-correlation with the appropriate template. The heliocentric RVs from FEROS and du Pont are reported in Tables 8 and 9, respectively. Typical uncertainties for FEROS are about 2.7 and $3.7 \mathrm{~km} \mathrm{~s}^{-1}$ for the primary and secondary of TWA $3 \mathrm{~A}$, respectively, and $3.3 \mathrm{~km} \mathrm{~s}^{-1}$ for TWA $3 \mathrm{~B}$. For the

\footnotetext{
${ }^{14}$ The slit width is smaller than the binary separation, resulting in varying amounts of light from stars A and B entering the slit at each observation depending on guiding and seeing.
} 
Table 8

FEROS Radial Velocity Measurements of TWA 3

\begin{tabular}{lccrr}
\hline \hline $\begin{array}{l}\text { HJD } \\
(2,400,000+)\end{array}$ & $\begin{array}{c}\text { Orbital } \\
\text { Phase }\end{array}$ & $\begin{array}{c}\mathrm{RV}_{\mathrm{Aa}} \\
\left(\mathrm{km} \mathrm{s}^{-1}\right)\end{array}$ & $\begin{array}{c}\mathrm{RV}_{\mathrm{Ab}} \\
\left(\mathrm{km} \mathrm{s}^{-1}\right)\end{array}$ & $\begin{array}{c}\mathrm{RV}_{\mathrm{B}} \\
\left(\mathrm{km} \mathrm{s}^{-1}\right)\end{array}$ \\
\hline $51,260.5221$ & 0.598 & 13.23 & 2.40 & 10.11 \\
$51,331.5915$ & 0.636 & 10.88 & -0.99 & 15.45 \\
$51,621.5378$ & 0.949 & 38.05 & -17.73 & 10.11 \\
$51,623.6000$ & 0.008 & 15.76 & 9.47 & 6.49 \\
$51,624.5913$ & 0.037 & -4.49 & 32.46 & 12.74 \\
$51,625.5186$ & 0.063 & -7.27 & 37.20 & 11.83 \\
$51,733.4665$ & 0.158 & -4.76 & 32.97 & 12.54 \\
$51,737.5133$ & 0.274 & -0.56 & 28.24 & 11.77 \\
\hline
\end{tabular}

Note. $\mathrm{RV}$ uncertainties are $\sigma_{\mathrm{Aa}}=2.61 \mathrm{~km} \mathrm{~s}^{-1}, \sigma_{\mathrm{Ab}}=3.59 \mathrm{~km} \mathrm{~s}^{-1}$, and $\sigma_{\mathrm{B}}=2.60 \mathrm{~km} \mathrm{~s}^{-1}$ (see text).

Table 9

Du Pont Radial Velocity Measurements of TWA 3

\begin{tabular}{lcccc}
\hline \hline $\begin{array}{l}\mathrm{HJD} \\
(2,400,000+)\end{array}$ & $\begin{array}{c}\text { Orbital } \\
\text { Phase }\end{array}$ & $\begin{array}{c}\mathrm{RV}_{\mathrm{Aa}} \\
\left(\mathrm{km} \mathrm{s}^{-1}\right)\end{array}$ & $\begin{array}{c}\mathrm{RV}_{\mathrm{Ab}} \\
\left(\mathrm{km} \mathrm{s}^{-1}\right)\end{array}$ & $\begin{array}{c}\mathrm{RV}_{\mathrm{B}} \\
\left(\mathrm{km} \mathrm{s}^{-1}\right)\end{array}$ \\
\hline $53,765.6694$ & 0.422 & 4.97 & 11.56 & 16.68 \\
$53,765.8423^{\mathrm{a}}$ & $\ldots$ & $\ldots$ & $\ldots$ & 10.56 \\
$53,765.8556^{\mathrm{b}}$ & 0.427 & 6.52 & 15.92 & $\ldots$ \\
$53,766.7060$ & 0.452 & 5.46 & 13.08 & 13.79 \\
$53,767.7278$ & 0.481 & 6.77 & 14.30 & 18.40 \\
$53,767.8467$ & 0.485 & 7.50 & 13.55 & 16.57 \\
$53,769.7425$ & 0.539 & 13.42 & 6.66 & 8.52 \\
$53,770.7760$ & 0.569 & 13.38 & 4.58 & 15.00 \\
$53,771.7166$ & 0.595 & 13.40 & 5.98 & 8.05 \\
\hline
\end{tabular}

Notes. RV uncertainties are $\sigma_{\mathrm{Aa}}=1.46 \mathrm{~km} \mathrm{~s}^{-1}, \sigma_{\mathrm{Ab}}=2.34 \mathrm{~km} \mathrm{~s}^{-1}$, and $\sigma_{\mathrm{B}}=3.95 \mathrm{~km} \mathrm{~s}^{-1}$ (see text).

${ }^{\mathrm{a}}$ Spectrum of TWA 3B alone.

b Spectrum of TWA 3A alone.

$\mathrm{du}$ Pont spectra the errors are 1.5, 2.4, and $2.7 \mathrm{~km} \mathrm{~s}^{-1}$, respectively. The light ratios between stars $\mathrm{Ab}$ and $\mathrm{Aa}$ determined from these spectra are $0.55 \pm 0.08$ for FEROS and $0.50 \pm 0.10$ for du Pont at a mean wavelength of $5190 \AA$, consistent with the measurement from the CfA spectra. The result from the du Pont light ratios is less reliable than the others because the du Pont observations were all made at epochs when both components were close to the center-of-mass velocity. The average of the CfA and FEROS light ratios, taken at favorable epochs, is $0.57 \pm 0.06$.

\section{Orbital Solutions}

\subsection{The TWA 3Aa-Ab Spectroscopic Binary}

Although the $\mathrm{Ab} / \mathrm{Aa}$ mass ratio is relatively large $(q=0.841$; see Table 10$)$, early analysis of the optical data sets did not yield a consistent solution, likely the result of the confusion introduced by the B component in the blended spectra (Section 3.2). Therefore, prior to combining all of the observations, we carried out an analysis of the 11 pairs of IR velocities alone (Table 6) using standard, nonlinear LevenbergMarquardt least-squares techniques (Press et al. 1992). This fit is shown in the second column of Table 10. With this initial solution as a guide, we were then able to obtain an independent solution for the CfA optical data (Table 7), with weights for the individual velocities inversely proportional to their uncertainties. This CfA-only solution is shown in the third column of Table 10 and shows good agreement with the previous fit, particularly for the velocity semiamplitudes that determine the minimum masses. The only exception is the center-of-mass velocity $\gamma$, for which the difference is likely the result of zeropoint uncertainties discussed below. Our du Pont spectra (which were scheduled before we had determined the ephemeris for TWA 3A) were obtained at very unfavorable orbital phases near conjunction and do not allow for the calculation of an independent orbital solution. The phase coverage and number of the FEROS measurements are also insufficient for a separate fit, but they do sample the velocity extremes. A constrained solution using the FEROS data with the ephemeris and geometric parameters ( $e$ and $\omega$ ) held fixed from the Keck results yields rough velocity amplitudes of $K_{\mathrm{Aa}}=21.9 \pm 2.4 \mathrm{~km} \mathrm{~s}^{-1}$ and $K_{\mathrm{Ab}}=29.1 \pm 2.6 \mathrm{~km} \mathrm{~s}^{-1}$, consistent with those from the Keck and CfA fits.

For the final solution we combined the four data sets, and in order to account for possible differences in velocity zeropoints, we solved for three offsets between each data set and the CfA set taken as the reference, as it has the most measurements. Additionally, because our analysis techniques do not return internal errors for the velocities, we made initial estimates of these for each data set based on the velocity scatter from preliminary solutions and then rescaled those errors by iterations in our global fit so as to achieve reduced $\chi^{2}$ values of unity, separately for each star and each data set. These final uncertainties are the ones reported in Tables 6-9. The orbital elements from our combined fit are listed in the last column of Table 10, along with other quantities. This solution is dominated by the near-IR velocities, which have the highest precision and a weight that is more than 5 times greater than the next most precise data set (du Pont). Nevertheless, the inclusion of the less precise data has improved the formal uncertainties of all elements. A graphical representation of our solution is shown in Figure 3, along with residuals for each data set.

Of the three velocity offsets reported in Table 10, only the one between CfA and Keck is statistically significant: $\Delta R V($ CfA-Keck $)=+1.53 \pm 0.43 \mathrm{~km} \mathrm{~s}^{-1}$. While this may be attributable in part to instrumental effects, we note that the velocities derived from the CfA, FEROS, and du Pont spectra (which seem to have consistent zero-points) all used the same templates, whereas those from Keck used different ones. It is likely, therefore, that the zero-point shift for Keck is the result of uncertainties in the RVs adopted for the templates.

We note that Malo et al. (2014) have recently published a few RV measurements of all three visible components of TWA 3. Their velocities for the B component agree well with ours, and although full dates were not provided for their measurements of $\mathrm{Aa}$ and $\mathrm{Ab}$, they too seem consistent with our spectroscopic orbital solution.

\subsection{The Visual Orbit of the Close TWA $3 A a-A b$ Binary}

The close pair TWA 3Aa-Ab was resolved spatially with the PIONIER combiner on the VLTI (Anthonioz et al. 2015) at a separation of $3.51 \pm 0.57$ mas and position angle of $108^{\circ} .1 \pm 9^{\circ} .3$ on HJD 2,455,601.870. We explored the range of orbital solutions consistent with this measurement by fixing the spectroscopic orbital parameters $(P, T, e, \omega)$ and selecting values for the angular semimajor axis, inclination, and longitude of the line of nodes $(a, i, \Omega)$ at random. We found that the $1 \sigma$ confidence intervals are well defined for $a$ (4.7-6.5 mas) and $\Omega\left(93^{\circ}-123^{\circ}\right)$, but that the confidence region for $i$ extends from $0^{\circ}$ to $180^{\circ}$. If we add the constraint that the 
Table 10

Spectroscopic Orbital Solutions for TWA 3A

\begin{tabular}{|c|c|c|c|}
\hline Parameter & Keck & CfA & Combined $^{\mathrm{a}}$ \\
\hline$P$ (days) & $34.8799 \pm 0.0022$ & $34.8742 \pm 0.0088$ & $34.87846 \pm 0.00090$ \\
\hline$\gamma\left(\mathrm{km} \mathrm{s}^{-1}\right)$ & $+8.63 \pm 0.18$ & $+9.61 \pm 0.41$ & $+10.17 \pm 0.40^{\mathrm{b}}$ \\
\hline$K_{\mathrm{Aa}}\left(\mathrm{km} \mathrm{s}^{-1}\right)$ & $23.38 \pm 0.36$ & $23.33 \pm 0.68$ & $23.28 \pm 0.26$ \\
\hline$e$ & $0.6323 \pm 0.0091$ & $0.636 \pm 0.019$ & $0.6280 \pm 0.0060$ \\
\hline$\omega_{\mathrm{Aa}}(\operatorname{deg})$ & $81.2 \pm 1.5$ & $76.5 \pm 2.6$ & $80.5 \pm 1.2$ \\
\hline$T(\mathrm{HJD}-2,400,000)^{\mathrm{c}}$ & $52,704.53 \pm 0.13$ & $52,704.24 \pm 0.20$ & $52,704.554 \pm 0.063$ \\
\hline$\Delta R V(\mathrm{CfA}-\mathrm{du}$ Pont $)\left(\mathrm{km} \mathrm{s}^{-1}\right)$ & $\cdots$ & $\cdots$ & $+0.15 \pm 0.61$ \\
\hline \multicolumn{4}{|c|}{ Derived Quantities } \\
\hline$M_{\mathrm{Aa}} \sin ^{3} i\left(M_{\odot}\right)$ & $0.1218 \pm 0.0055$ & $0.117 \pm 0.010$ & $0.1224 \pm 0.0042$ \\
\hline$M_{\mathrm{Ab}} \sin ^{3} i\left(M_{\odot}\right)$ & $0.1027 \pm 0.0043$ & $0.0999 \pm 0.0071$ & $0.1030 \pm 0.0032$ \\
\hline$q \equiv M_{\mathrm{Ab}} / M_{\mathrm{Aa}}$ & $0.843 \pm 0.018$ & $0.851 \pm 0.040$ & $0.841 \pm 0.014$ \\
\hline \multicolumn{4}{|c|}{ Other Quantities Pertaining to the Fit } \\
\hline$N_{\mathrm{Aa}}, N_{\mathrm{Ab}}$, Keck & 11,11 & $\cdots$ & 11,11 \\
\hline$N_{\mathrm{Aa}}, N_{\mathrm{Ab}}, \mathrm{CfA}$ & $\cdots$ & 35,35 & 35,35 \\
\hline$N_{\mathrm{Aa}}, N_{\mathrm{Ab}}$, FEROS & $\cdots$ & $\cdots$ & 8,8 \\
\hline$N_{\mathrm{Aa}}, N_{\mathrm{Ab}}, \mathrm{du}$ Pont & $\cdots$ & $\cdots$ & 8,8 \\
\hline Time span (days) & 2970.8 & 3308.0 & 4773.0 \\
\hline$\sigma_{\mathrm{Aa}}, \sigma_{\mathrm{Ab}}, \operatorname{Keck}\left(\mathrm{km} \mathrm{s}^{-1}\right)$ & $0.73,1.05$ & $\ldots$ & $0.66,0.89$ \\
\hline$\sigma_{\mathrm{Aa}}, \sigma_{\mathrm{Ab}}$, CfA $\left(\mathrm{km} \mathrm{s}^{-1}\right)$ & $\ldots$ & $2.74,4.64$ & $2.74,4.45$ \\
\hline$\sigma_{\mathrm{Aa}}, \sigma_{\mathrm{Ab}}, \operatorname{FEROS}\left(\mathrm{km} \mathrm{s}^{-1}\right)$ & $\cdots$ & $\ldots$ & $2.72,3.74$ \\
\hline$\sigma_{\mathrm{Aa}}, \sigma_{\mathrm{Ab}}$, du Pont $\left(\mathrm{km} \mathrm{s}^{-1}\right)$ & $\cdots$ & $\cdots$ & $1.52,2.45$ \\
\hline
\end{tabular}

Notes. $\omega_{\mathrm{Ab}}=\omega_{\mathrm{Aa}}+180=260^{\circ} .5$.

${ }^{a}$ Simultaneous solution using the Keck, CfA, FEROS, and du Pont data sets.

${ }^{\mathrm{b}}$ On the reference system of CfA.

${ }^{c}$ Time of periastron passage nearest to the average of all times of observation from the four data sets.

orbital parallax (measured from the $K_{1}, K_{2}, P, a, e$, and $i$ ) lies within the uncertainties of the parallax derived from the moving group cluster method ( $d=34 \pm 4 \mathrm{pc}$; Mamajek 2005), then we find that the allowable ranges for $i$ fall into two families of solutions between $32^{\circ}$ and $63^{\circ}$ and between $118^{\circ}$ and $149^{\circ}$, as shown in Figure 4 . These ranges yield absolute masses of $M_{1}$ from 0.17 to $0.86 M_{\odot}$ and $M_{2}$ from 0.14 to $0.72 M_{\odot}$.

Adopting a value for the angular semimajor axis, 5.6 mas, that falls in the average of the range of well-defined confidence intervals, 4.7-6.5 mas, and assuming a distance of $34 \mathrm{pc}$ (Mamajek 2005), we estimate the physical size of the semimajor axis to be $\sim 0.19$ au. Given the eccentricity of the $\mathrm{SB}$ orbit, 0.628 , we find an orbital periastron distance of 0.07 au and an apastron distance of 0.31 au.

\subsection{The Visual Orbit of the Wide TWA 3A-B Binary}

We measured the relative RVs between the four epochs of observation of TWA 3B shown in Figure 2 by cross-correlating the highest signal-to-noise spectrum, from UT 2002 December 22, against all other epochs. The RVs of TWA 3B appear constant, within the $\sim 1 \mathrm{~km} \mathrm{~s}^{-1}$ uncertainties; thus, there is no evidence to indicate that TWA 3B is also a spectroscopic binary. The measured difference between the RV of TWA $3 \mathrm{~B}$ and the center-of-mass velocity of TWA $3 \mathrm{~A}$ is $-1.21 \pm 0.25 \mathrm{~km} \mathrm{~s}^{-1}$ from the Keck observations and $-0.73 \pm 0.60 \mathrm{~km} \mathrm{~s}^{-1}$ from the
CfA velocities, consistent with a physical association between the two visual components.

To investigate the properties of the TWA $3 \mathrm{~A}-\mathrm{B}$ orbit, we downloaded measurements of the wide binary separation from the Washington Double Star Catalog ${ }^{15}$ (Mason et al. 2001). From this catalog, we used eight epochs from 1992 through 2015 to investigate the relative motion between this pair of stars (Reipurth \& Zinnecker 1993; Webb et al. 1999; Weintraub et al. 2000; Brandeker et al. 2003; Correia et al. 2006; Janson et al. 2014; Tokovinin et al. 2015; B.D. Mason et al. 2017, in preparation). We supplemented these data with observations made at the DCT (Section 2.2.2) and Magellan (Section 2.1.2) telescopes (Table 4). For the measurement reported by Reipurth \& Zinnecker (1993) we used an observation date of 1992 January 8/9 (Besselian Year 1992.0216), supplied by B. Reipurth (2015, private communication).

The relative motion between TWA $3 \mathrm{~A}$ and 3B covers only a small arc (Figure 5). We computed a linear least-squares fit to measure the relative motion between the components in

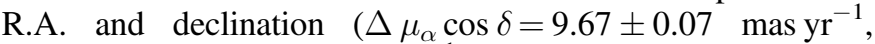

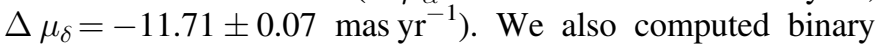
orbit fits using the grid search procedure described in Schaefer et al. (2014), but we found that the $\chi^{2}$ from the orbit fit was indistinguishable from the linear fit. To determine realistic

\footnotetext{
15 http://ad.usno.navy.mil/wds
} 


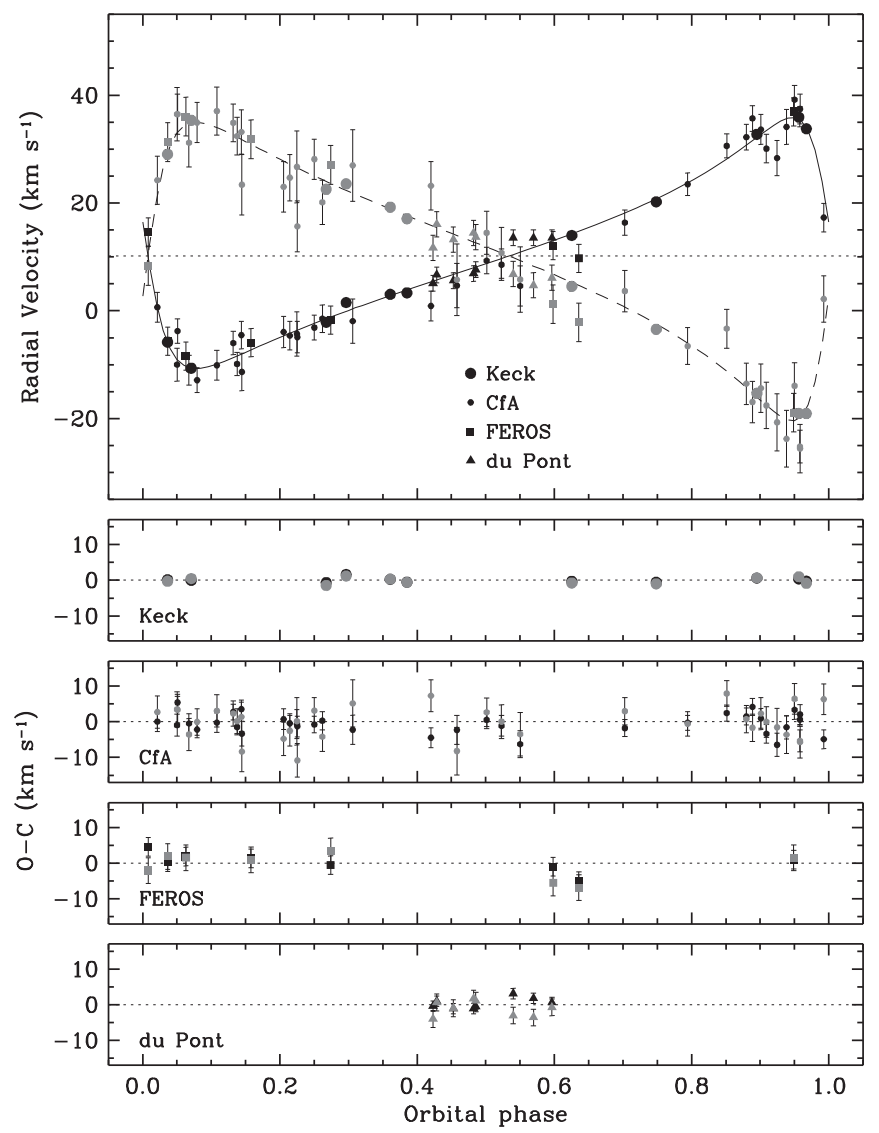

Figure 3. Top: RVs for TWA $3 \mathrm{~A}$ and our model from the best-fit solution of Table 10 (solid line for the primary, dashed line for the secondary). The dotted line indicates the center-of-mass velocity of the system. Measurements from different data sets are represented with different symbols, as labeled. Bottom panels: velocity residuals $(O-C)$ shown separately for each data set with the same error bars as in the top panel.

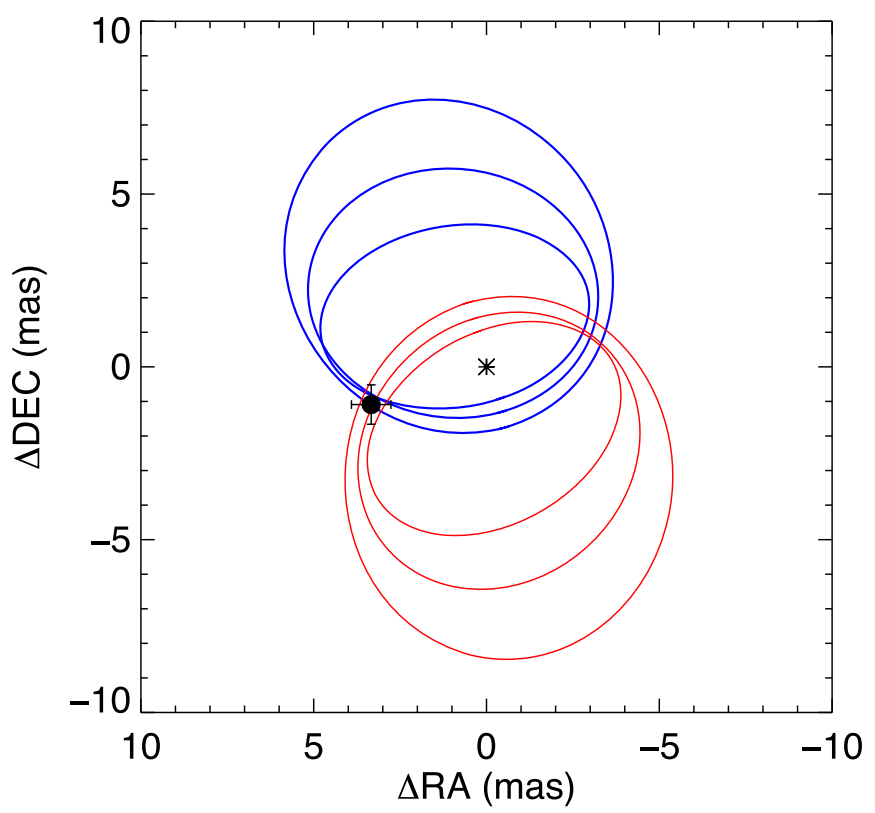

Figure 4. Sample orbits consistent with the $1 \sigma$ uncertainties from the VLTI PIONIER separation measurement of TWA $3 \mathrm{Aa}-\mathrm{Ab}$ (filled black circle). These orbits were found by fixing the spectroscopic orbital parameters and selecting values for $a, i$, and $\Omega$ at random. If we add a constraint that the orbital parallax lies within $d=34 \pm 4$ pc (Mamajek 2005), then we find that the orbits cluster into two groups with $i<90^{\circ}$ (red orbits) and $i>90^{\circ}$ (blue orbits).

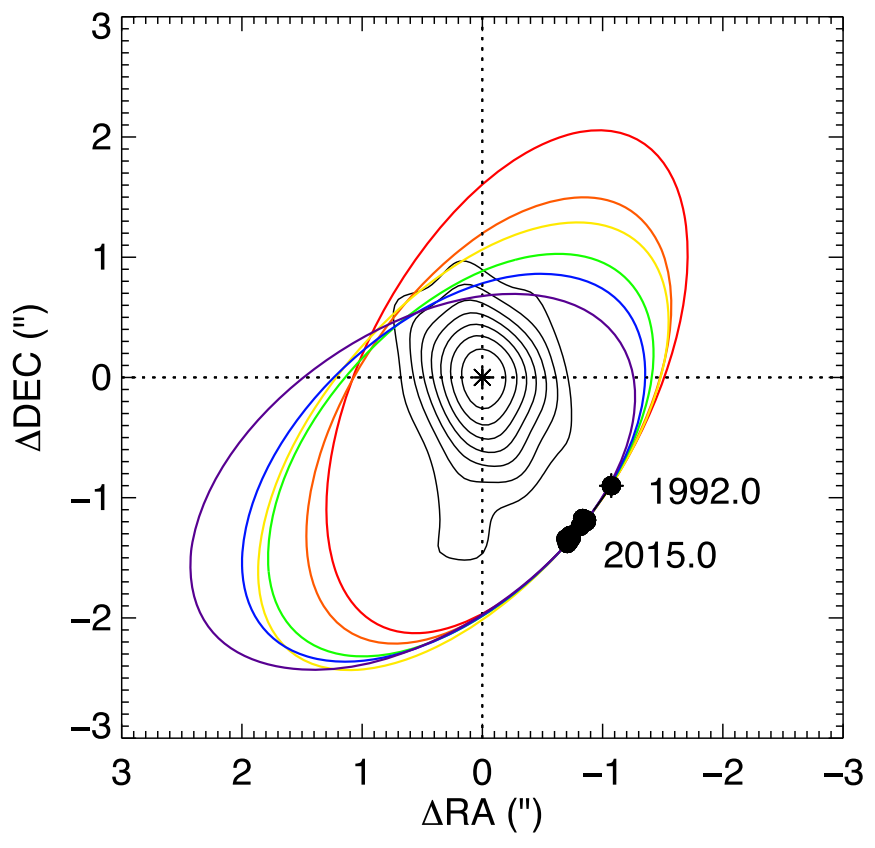

Figure 5. Motion of TWA 3B relative to TWA 3A from observations listed in the Washington Double Star Catalog and the Magellan and DCT observations. The colored lines show examples of orbits that fit the data and produce a total mass in the range of $0.79-0.81 M_{\odot}$, assuming a distance of $34 \mathrm{pc}$. The parameters for the plotted orbits are listed in Table 12. The contours show the $880 \mu \mathrm{m}$ dust continuum emission from the disk around TWA 3A, convolved with the beam size. These are plotted in $2 \sigma$ intervals, beginning at $3 \sigma(10 \mathrm{mJy}$ beam ${ }^{-1}$; from Andrews et al. 2010).

Table 11

Range of Orbital Parameters Obtained for Wide A-B Orbit Search

\begin{tabular}{lcc}
\hline \hline Parameter & Minimum & Maximum \\
\hline$P(\mathrm{yr})$ & 236 & 800 \\
$T(\mathrm{BY})$ & 1610 & 2386 \\
$e$ & 0.00 & 0.80 \\
$a(\operatorname{arcsec})$ & 1.17 & 3.18 \\
$i(\mathrm{deg})$ & 108 & 172 \\
$\Omega(\mathrm{deg})$ & 0 & 140 \\
$\cdots$ & 320 & 360 \\
$\omega_{B}(\operatorname{deg})$ & 0 & 360 \\
$M\left(M_{\odot}\right)$ & 0.32 & 2.00 \\
\hline
\end{tabular}

Note. While searching for orbital solutions, we added a constraint that the total mass must be smaller than $2 M_{\odot}$, assuming a distance of $34 \mathrm{pc}$. We placed an arbitrary upper limit of $800 \mathrm{yr}$ on the period.

ranges for the orbital parameters, we randomly searched the parameter space and added a constraint that the total system mass, assuming a distance of $34 \mathrm{pc}$ (Mamajek et al. 2005), must be less than $2.0 M_{\odot}$. This is roughly double the expected total mass of $0.8 M_{\odot}$ based on the component spectral types and the $10 \mathrm{Myr}$ evolutionary tracks computed from Baraffe et al. (2015). Table 11 shows the ranges of orbital parameters for solutions obtained in our random grid search procedure. We show six example orbits in Figure 5 with masses in the range of $0.78-0.81 M_{\odot}$ and inclinations ranging from $120^{\circ}$ to $128^{\circ}$. The parameters for the selected orbits are given in Table 12; these orbits are not definitive and should only be used as a way to estimate the range of possible motion in the near future.

The concordance of position, proper motion, and RV all are consistent with TWA 3A and 3B being a bound pair. To assess 
Table 12

Parameters for Wide A-B Orbits Plotted in Figure 5

\begin{tabular}{lcccccc}
\hline \hline $\begin{array}{l}P \\
(\mathrm{yr})\end{array}$ & $\begin{array}{c}T \\
(\mathrm{BY})\end{array}$ & $e$ & $\begin{array}{c}a \\
(\operatorname{arcsec})\end{array}$ & $\begin{array}{c}i \\
(\mathrm{deg})\end{array}$ & $\begin{array}{c}\Omega \\
(\mathrm{deg})\end{array}$ & $\begin{array}{c}\omega_{B} \\
(\mathrm{deg})\end{array}$ \\
\hline 785.76 & 2321.3 & 0.1694 & 2.321 & 120.67 & 331.95 & 260.46 \\
$\left(M_{\odot}\right)$
\end{tabular}

Note. The total masses were computed assuming a distance of $34 \mathrm{pc}$ from Mamajek (2005).

the likelihood that TWA 3A and 3B are gravitationally bound to each other, we compared the total spatial velocity as determined from RV and proper-motion measurements with the escape velocity for TWA 3A. We estimated the combined mass of the TWA $3 \mathrm{~A}$ binary at $0.37 M_{\odot}$, based on our spectral classification. In the more conservative estimate, the difference in $\mathrm{RV}$ between the $3 \mathrm{~A}$ center of mass and $3 \mathrm{~B}$ is $-1.21 \mathrm{~km} \mathrm{~s}^{-1}$.

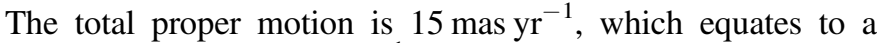
space velocity of $2.4 \mathrm{~km} \mathrm{~s}^{-1}$, given a total velocity difference of $2.7 \mathrm{~km} \mathrm{~s}^{-1}$ between $\mathrm{A}$ and $\mathrm{B}$. The escape velocity from $3 \mathrm{~A}$ is $\sqrt{2 G M_{\mathrm{A}} / r_{\mathrm{AB}}}$, which equals $3.6 \mathrm{~km} \mathrm{~s}^{-1}$ in the case where the separation between the components is purely in the sky plane, $r_{\mathrm{AB}} \sim 50 \mathrm{au}$. If we estimate an additional separation in the lineof-sight direction equal to the sky-plane separation, the escape velocity drops to $3.0 \mathrm{~km} \mathrm{~s}^{-1}$. In either case, $3 \mathrm{~A}$ and $3 \mathrm{~B}$ can be plausibly argued to be bound to each other.

Finally, the H-R diagram displayed in Figure 6 shows that all three components are consistent with a $10 \mathrm{Myr}$ age to within our uncertainties. We used values for $T_{\text {eff }}$ determined for our best-fit RV template spectra (Section 3) by Mann et al. (2015). The optical solutions used GJ 48 (M3) to fit the B component and GJ 699 (M4) for both stars in the A component. In the IR we found the best fits with GJ 15A (M3; Prato 2007) for the B component, GJ 402 (M4) for the Aa component, and GJ 669B (M4.5) for the $\mathrm{Ab}$ component. As both the IR and optical solutions used an M3 for the B component, we determined the average $T_{\text {eff }}$ for the two M3 stars with metallicity closest to solar given in Table 5 of Mann et al. and propagated their uncertainties, yielding $T_{\text {eff }}(\mathrm{B})=3453 \pm 86 \mathrm{~K}$. Although Mann et al. provide a $T_{\text {eff }}$ estimate for GJ $15 \mathrm{~A}$, they do not for GJ 48. Prato (2007) indicates an M3 type for GJ 15A; however, Mann et al. found an earlier type of M1.4. For the primary star in the spectroscopic binary, both the optical and IR solutions indicate an M4. Mann et al. give $T_{\text {eff }}=3238 \pm 60 \mathrm{~K}$ for GJ 402, close to the $3228 \pm 60 \mathrm{~K}$ value for GJ 699 . We used the former $T_{\text {eff. }}$. For the spectroscopic binary secondary, Mann et al. do not give a $T_{\text {eff }}$ for GJ 669B, so we again used the average of the two M4.5 stars in their Table 5 with metallicity closest to solar and found $T_{\text {eff }}=3131 \pm 85 \mathrm{~K}$. We calculated absolute H-band magnitudes using the $\mathrm{A}$ and $\mathrm{B}$ component magnitudes from Webb et al. (1999) given in Table 13. To determine the apparent $\mathrm{H}$ magnitudes of the $\mathrm{Aa}$ and $\mathrm{Ab}$ components, we used the value for TWA 3A from Webb et al. in combination with the average spectroscopic binary $H$-band flux ratio, $0.61 \pm 0.11$, found from cross-correlation of nine epochs of IR spectroscopy (Section 3.1). We measured the absolute $H$-band magnitudes using $d=34 \pm 4 \mathrm{pc}$ (Mamajek 2005). The coevality of the three components does not necessarily imply that they formed together, but the lack of

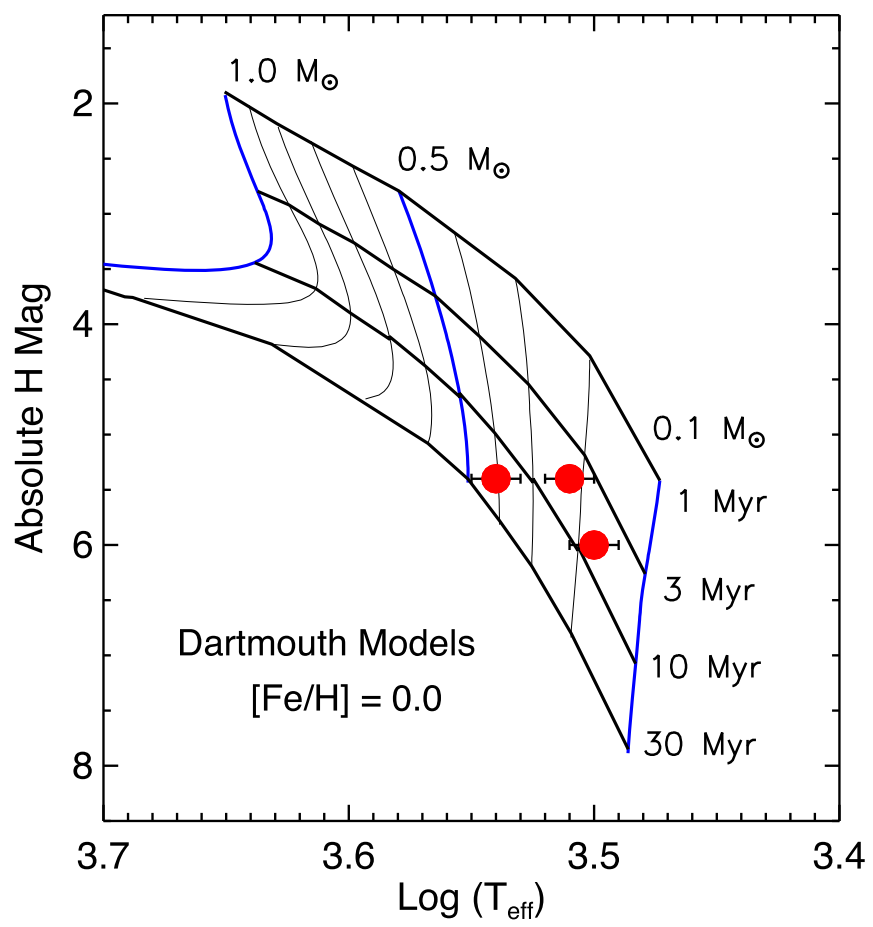

Figure 6. H-R diagram for all three TWA 3 components. Mass tracks and isochrones of the solar-metallicity Dartmouth evolutionary tracks (Dotter et al. 2008) are labeled. See text for further details. The uncertainties in absolute $\mathrm{H}$ magnitude are all $0.1 \mathrm{mag}$, smaller than the plotted points. All three components' positions are consistent with an age of $10 \mathrm{Myr}$.

a discrepancy in age is in any case a requirement for a common origin. We note that the mass ratio of $0.841 \sim \pm 0.014$ that we determine for the orbit of TWA Aa-Ab (Table 10) is consistent with the ratio of the mass tracks on which these components fall, to within $1 \sigma$, in the HR diagram (Figure 6).

\section{The Distribution of Stars, Gas, and Dust in the TWA 3 System}

TWA 3 has been historically characterized as a CTTS, i.e., a disk-bearing system. De la Reza et al. (1989) identified the wide A-B binary for the first time "at the Coude focus" when they first observed TWA 3. They determined that at least one of the TWA 3 components was associated with the coincident IRAS source, implying the presence of warm dust, and found $\mathrm{H} \alpha$ equivalent widths of 20 and $8 \AA$ for the $\mathrm{A}$ and $\mathrm{B}$ components, respectively, which they interpreted to imply that both $\mathrm{A}$ and $\mathrm{B}$ were actively accreting circumstellar gas; this interpretation has not been born out for the B component in subsequent observations, and it appears to be diskless. 
Table 13

TWA 3 Magnitudes and Flux Densities

\begin{tabular}{|c|c|c|c|c|c|c|c|}
\hline Filter & $\begin{array}{c}\lambda \\
(\mu \mathrm{m})\end{array}$ & $\begin{array}{c}\mathrm{A} \\
(\mathrm{mag})\end{array}$ & $\begin{array}{c}\mathrm{B} \\
(\mathrm{mag})\end{array}$ & $\begin{array}{c}\mathrm{A}+\mathrm{B} \\
(\mathrm{mag})\end{array}$ & $\begin{array}{c}\lambda \mathrm{F}_{\lambda} \mathrm{A} \\
\left(\mathrm{erg} \mathrm{cm}^{-2} \mathrm{~s}^{-1}\right)\end{array}$ & $\begin{array}{c}\lambda \mathrm{F}_{\lambda} \mathrm{B} \\
\left(\mathrm{erg} \mathrm{cm}^{-2} \mathrm{~s}^{-1}\right)\end{array}$ & Source \\
\hline$U$ & 0.3656 & $\cdots$ & $\cdots$ & 14.05 & $\cdots$ & $\cdots$ & 1 \\
\hline$U$ & 0.3656 & $\cdots$ & $\cdots$ & 14.21 & $\cdots$ & $\cdots$ & 2 \\
\hline$U$ & 0.3656 & $\cdots$ & $\cdots$ & 14.27 & $\cdots$ & $\cdots$ & 3 \\
\hline$B$ & 0.4353 & $\cdots$ & $\cdots$ & $13.537 \pm 0.02$ & $\ldots$ & $\ldots$ & 4 \\
\hline$B$ & 0.4353 & $\cdots$ & $\cdots$ & 13.58 & $\cdots$ & $\cdots$ & 1 \\
\hline$B$ & 0.4353 & $\ldots$ & $\cdots$ & 13.52 & $\ldots$ & $\cdots$ & 2 \\
\hline$B$ & 0.4353 & $\ldots$ & $\ldots$ & 13.53 & $\ldots$ & $\ldots$ & 3 \\
\hline $\boldsymbol{B}$ & 0.44 & 14.04 & 14.59 & $\ldots$ & $7.669 \times 10^{-11}$ & $4.621 \times 10^{-11}$ & 5 \\
\hline$g^{\prime}$ & 0.4639 & $\ldots$ & $\ldots$ & $12.74 \pm 0.02$ & $\ldots$ & $\ldots$ & 4 \\
\hline$V$ & 0.5477 & $\cdots$ & $\cdots$ & $12.05 \pm 0.01$ & $\cdots$ & $\cdots$ & 4 \\
\hline$V$ & 0.5477 & $\cdots$ & $\cdots$ & 12.06 & $\cdots$ & $\cdots$ & 1 \\
\hline$V$ & 0.5477 & $\cdots$ & $\cdots$ & 12.04 & $\cdots$ & $\cdots$ & 2 \\
\hline$V$ & 0.5477 & $\cdots$ & $\cdots$ & 12.04 & $\cdots$ & $\cdots$ & 3 \\
\hline$V$ & 0.55 & 12.57 & 13.07 & $\ldots$ & $2.022 \times 10^{-10}$ & $1.276 \times 10^{-10}$ & 5 \\
\hline$r^{\prime}$ & 0.6122 & $\ldots$ & $\cdots$ & $11.442 \pm 0.02$ & $\ldots$ & $\ldots$ & 4 \\
\hline $\mathrm{Rc}$ & 0.6407 & $\cdots$ & $\cdots$ & 10.72 & $\cdots$ & $\cdots$ & 1 \\
\hline $\mathrm{Rc}$ & 0.6407 & $\cdots$ & $\cdots$ & 10.66 & $\cdots$ & $\cdots$ & 2 \\
\hline $\mathrm{Rc}$ & 0.6407 & $\cdots$ & $\cdots$ & 10.69 & $\cdots$ & $\cdots$ & 3 \\
\hline $\mathbf{R c}^{\mathrm{a}}$ & 0.6407 & $11.28 \pm 0.01$ & $\mathbf{1 1 . 6 3} \pm \mathbf{0 . 0 1}$ & $\ldots$ & $4.141 \times 10^{-10}$ & $3.000 \times 10^{-10}$ & $1,2,3,6$ \\
\hline$i^{\prime}$ & 0.7439 & $\ldots$ & $\ldots$ & $9.826 \pm 0.05$ & $\ldots$ & $\ldots$ & 4 \\
\hline$I$ & 0.79 & $\cdots$ & $\cdots$ & $9.29 \pm 0.03$ & $\cdots$ & $\cdots$ & 7 \\
\hline$I$ & 0.79 & 9.72 & 10.0 & $\ldots$ & $1.101 \times 10^{-09}$ & $8.510 \times 10^{-10}$ & 5 \\
\hline Ic & 0.798 & $\cdots$ & $\cdots$ & 9.14 & $\cdots$ & $\cdots$ & 1 \\
\hline Ic & 0.798 & $\cdots$ & $\cdots$ & 9.11 & $\cdots$ & $\cdots$ & 2 \\
\hline Ic & 0.798 & $\cdots$ & $\cdots$ & 9.1 & $\cdots$ & $\cdots$ & 3 \\
\hline $\mathbf{I} \mathbf{c}^{\mathrm{a}}$ & 0.798 & $9.69 \pm 0.01$ & $10.08 \pm 0.01$ & $\cdots$ & $1.121 \times 10^{-09}$ & $1.004 \times 10^{-09}$ & $1,2,3,6$ \\
\hline$J$ & 1.21 & $8.22 \pm 0.10$ & $8.63 \pm 0.10$ & $\ldots$ & $2.046 \times 10^{-09}$ & $1.403 \times 10^{-09}$ & 8 \\
\hline$J$ & 1.21 & $8.25 \pm 0.03$ & $8.69 \pm 0.03$ & $\ldots$ & $1.991 \times 10^{-09}$ & $1.327 \times 10^{-09}$ & 9 \\
\hline$J$ & 1.21 & $\ldots$ & $\cdots$ & 7.61 & $\cdots$ & $\cdots$ & 10 \\
\hline$J$ & 1.235 & $\cdots$ & $\cdots$ & $7.651 \pm 0.019$ & $\cdots$ & $\cdots$ & 11 \\
\hline$J$ & 1.24 & $\ldots$ & $\ldots$ & $7.63 \pm 0.05$ & $\ldots$ & $\ldots$ & 7 \\
\hline F164N & 1.65 & $7.52 \pm 0.01$ & $7.9 \pm 0.01$ & $\ldots$ & $1.790 \times 10^{-09}$ & $1.2623 \times 10^{-09}$ & 12 \\
\hline $\boldsymbol{H}$ & 1.648 & $7.53 \pm 0.05$ & $8.15 \pm 0.07$ & $\cdots$ & $1.788 \times 10^{-09}$ & $1.010 \times 10^{-09}$ & 13 \\
\hline $\boldsymbol{H}$ & 1.65 & $7.6 \pm 0.1$ & $8.1 \pm 0.1$ & $\ldots$ & $1.675 \times 10^{-09}$ & $1.086 \times 10^{-09}$ & 8 \\
\hline $\boldsymbol{H}$ & 1.65 & $7.79 \pm 0.03$ & $8.19 \pm \mathbf{0 . 0 3}$ & $\cdots$ & $1.406 \times 10^{-09}$ & $9.727 \times 10^{-10}$ & 9 \\
\hline$H$ & 1.662 & $\ldots$ & $\ldots$ & $7.041 \pm 0.027$ & $\ldots$ & $\ldots$ & 11 \\
\hline F190N & 1.9 & $7.60 \pm 0.01$ & $7.98 \pm 0.01$ & $\ldots$ & $1.204 \times 10^{-09}$ & $8.488 \times 10^{-10}$ & 12 \\
\hline F215N & 2.15 & $7.37 \pm 0.01$ & $7.79 \pm 0.01$ & $\cdots$ & $1.055 \times 10^{-09}$ & $7.163 \times 10^{-10}$ & 12 \\
\hline$K$ & 2.159 & $\ldots$ & $\ldots$ & $6.77 \pm 0.02$ & $\ldots$ & $\ldots$ & 11 \\
\hline$K$ & 2.16 & $\ldots$ & $\ldots$ & $6.77 \pm 0.08$ & $\ldots$ & $\ldots$ & 7 \\
\hline$K$ & 2.2 & $7.28 \pm 0.07$ & $7.80 \pm 0.07$ & $\ldots$ & $1.052 \times 10^{-09}$ & $6.517 \times 10^{-10}$ & 8 \\
\hline$K$ & 2.2 & $\ldots$ & $\ldots$ & 6.73 & $\ldots$ & $\ldots$ & 10 \\
\hline$K$ & 2.2 & $7.39 \pm \mathbf{0 . 0 3}$ & $7.82 \pm \mathbf{0 . 0 3}$ & $\ldots$ & $9.507 \times 10^{-10}$ & $6.398 \times 10^{-10}$ & 9 \\
\hline W1 & 3.35 & $\ldots$ & $\ldots$ & $6.601 \pm 0.038$ & $\ldots$ & $\ldots$ & 14 \\
\hline$L$ & 3.45 & $7.05 \pm 0.08$ & $7.6 \pm \mathbf{0 . 0 8}$ & $\ldots$ & $3.659 \times 10^{-10}$ & $2.205 \times 10^{-10}$ & 9 \\
\hline Spitzer & 3.6 & $\cdots$ & $\cdots$ & $6.49 \pm 0.02$ & $\cdots$ & $\cdots$ & 15 \\
\hline Spitzer & 4.5 & $\ldots$ & $\ldots$ & $6.37 \pm 0.02$ & $\ldots$ & $\ldots$ & 15 \\
\hline W2 & 4.6 & $\cdots$ & $\cdots$ & $6.341 \pm 0.021$ & $\cdots$ & $\cdots$ & 14 \\
\hline$M$ & 4.8 & $6.8 \pm 0.1$ & $\cdots$ & $\ldots$ & $1.822 \times 10^{-10}$ & $\cdots$ & 9 \\
\hline Spitzer & 5.8 & $\cdots$ & $\cdots$ & $6.15 \pm 0.03$ & $\ldots$ & $\ldots$ & 15 \\
\hline Spitzer & 8.0 & $\ldots$ & $\cdots$ & $5.15 \pm 0.03$ & $\ldots$ & $\cdots$ & 15 \\
\hline$N$ & 10 & $\cdots$ & $\cdots$ & $\ldots$ & $2.012 \times 10^{-10}$ & $\cdots$ & 16 \\
\hline$N$ & 10.7 & $\cdots$ & $\ldots$ & $\cdots$ & $2.523 \times 10^{-10}$ & $<1.402 \times 10^{-11}$ & 17 \\
\hline W3 & 11.6 & $\cdots$ & $\cdots$ & $3.876 \pm 0.016$ & $\ldots$ & $\ldots$ & 14 \\
\hline IRAS & 12 & $\cdots$ & $\cdots$ & $\ldots$ & $2.471 \times 10^{-10 b}$ & $\cdots$ & 18 \\
\hline$Q$ & 18.2 & $\cdots$ & $\cdots$ & $\cdots$ & $8.242 \times 10^{-11}$ & $\cdots$ & 17 \\
\hline W4 & 22.1 & $\cdots$ & $\cdots$ & $1.734 \pm 0.014$ & $\cdots$ & $\cdots$ & 14 \\
\hline Spitzer & 24.0 & $\cdots$ & $\cdots$ & $1.62 \pm 0.04$ & $\cdots$ & $\cdots$ & 15 \\
\hline IRAS & 25 & $\cdots$ & $\cdots$ & $\cdots$ & $2.096 \times 10^{-10 b}$ & $\cdots$ & 18 \\
\hline IRAS & 60 & $\cdots$ & $\cdots$ & $\cdots$ & $4.597 \times 10^{-11 b}$ & $\ldots$ & 18 \\
\hline IRAS & 100 & $\cdots$ & $\cdots$ & $\ldots$ & $3.543 \times 10^{-11 b}$ & $\ldots$ & 18 \\
\hline Far-IR & 100 & $\ldots$ & $\ldots$ & $\ldots$ & $5.600 \times 10^{-12 b}$ & $\ldots$ & 19 \\
\hline Far-IR & 160 & $\cdots$ & $\ldots$ & $\ldots$ & $2.869 \times 10^{-12 b}$ & $\ldots$ & 19 \\
\hline
\end{tabular}


Table 13

(Continued)

\begin{tabular}{lcccccc}
\hline \hline Filter & $\begin{array}{c}\lambda \\
(\mu \mathrm{m})\end{array}$ & $\begin{array}{c}\mathrm{A} \\
(\mathrm{mag})\end{array}$ & $\begin{array}{c}\mathrm{B} \\
(\mathrm{mag})\end{array}$ & $\begin{array}{c}\mathrm{A}+\mathrm{B} \\
(\mathrm{mag})\end{array}$ & $\begin{array}{c}\lambda \mathrm{F}_{\lambda} \mathrm{A} \\
\left(\mathrm{erg} \mathrm{cm}^{-2} \mathrm{~s}^{-1}\right)\end{array}$ & $\begin{array}{c}\lambda \mathrm{F}_{\lambda} \mathrm{B} \\
\left(\mathrm{erg} \mathrm{cm}^{-2} \mathrm{~s}^{-1}\right)\end{array}$ \\
\hline Submillimeter & $\mathbf{8 8 0}$ & $\cdots$ & $\cdots$ & $\cdots$ & $\mathbf{8 . 5 2 3} \times \mathbf{1 0}^{-\mathbf{1 4 b}}$ & $\cdots$ \\
\hline
\end{tabular}

Notes.

${ }^{\mathrm{a}} \mathrm{Rc}$ and Ic magnitudes in boldface, plotted in Figure 7, were determined from the average of the Rc and Ic magnitudes from the literature in conjunction with the flux ratios for the $R$ and $I$ filters given in Table 4.

${ }^{\mathrm{b}}$ Unresolved flux densities for A + B; the majority of flux, an order of magnitude or more, is from the A component and so is attributed to A in the SED plots, top panel of Figure 7.

References. (1) de la Reza et al. 1989; (2) Gregorio-Hetem et al. 1992; (3) Torres et al. 2000; (4) Munari et al. 2014; (5) Torres et al. 2006; (6) this work; (7) Leggett et al. 2001; (8) Webb et al. 1999; (9) Geoffray \& Monin 2001; (10) Zuckerman 2001; (11) Cutri et al. 2003; (12) Weintraub et al. 2000; (13) Brandeker et al. 2003; (14) Cutri et al. 2012; (15) Luhman et al. 2010; (16) Metchev et al. 2004; (17) Jayawardhana et al. 1999; (18) Beichman et al. 1988; (19) Riviere-Marichalar et al. 2013; (20) Andrews et al. 2010.

Webb et al. (1999) comment in their Table 1 that TWA 3A is a candidate spectroscopic binary; Muzerolle et al. (2000) and Torres et al. (2003) provided additional evidence for this shortperiod pair. With our subsequent high-resolution IR spectroscopy campaign we demonstrate that TWA 3A, the "primary" star, is actually two later-type stars in a 35-day period orbit (Section 4.1). Jayawardhana et al. (1999) showed that the midIR excess, implying the presence of warm dust, is attributable to only the A component. Andrews et al. (2010) resolved the TWA 3A circumbinary disk at submillimeter wavelengths. We present evidence for an accretion disk in the TWA 3 system in the form of updated SEDs and hydrogen emission line equivalent widths, demonstrate that the circumbinary disk around TWA $3 \mathrm{~A}$ is not aligned with either stellar binary orbital plane, and examine the possible capture of TWA 3B as a potential formation mechanism for this system.

\subsection{SEDs and Accretion: Dust and Gas in TWA 3}

Figure 7 shows the individual SEDs for TWA 3A and TWA 3B. Although SEDs for this system have appeared elsewhere (e.g., Andrews et al. 2010), we provide specific literature sources and fluxes (Table 13) for future reference. Zuckerman (2001) suggested that the entire TWA 3 system was surrounded by cool, large grains; however, Andrews et al. found no evidence for this material. All excess IR and submillimeter flux is localized in a dusty circumbinary gas disk around the TWA $3 \mathrm{~A}$ component only, indicated by the mid-IR and longerwavelength points in Figure 7. Either TWA 3B was not detected at longer wavelengths, or the two components were too blended for individual flux densities to be determined. However, given that the one upper limit given for the $\mathrm{B}$ component at $10 \mu \mathrm{m}$ is $18 \times$ fainter than the flux density of TWA $3 \mathrm{~A}$ at that wavelength, it is safe to assume that the vast bulk of the longer-wavelength emission originates in the TWA 3 A circumbinary disk.

Andrews et al. (2010) modeled the resolved submillimeter flux and provided best-fit parameters for the disk, including an outer disk radius of 15-25 au and an inner radius for the dense disk of 1 au. In their model, material $500 \times$ more tenuous extends down to 0.4 au. The TWA $3 \mathrm{Aa}-\mathrm{Ab}$ probable family of orbits described in Section 4.2 and shown in Figure 4 has a range of semimajor axes of 4.7-6.5 mas. For an average value of 5.6 mas and the distance to TWA 3 of $34 \mathrm{pc}$, the corresponding physical separation of 0.19 au is consistent with the clearance of a small central hole in the disk model described by Andrews et al. (2010). Muzerolle et al. (2000) describe their
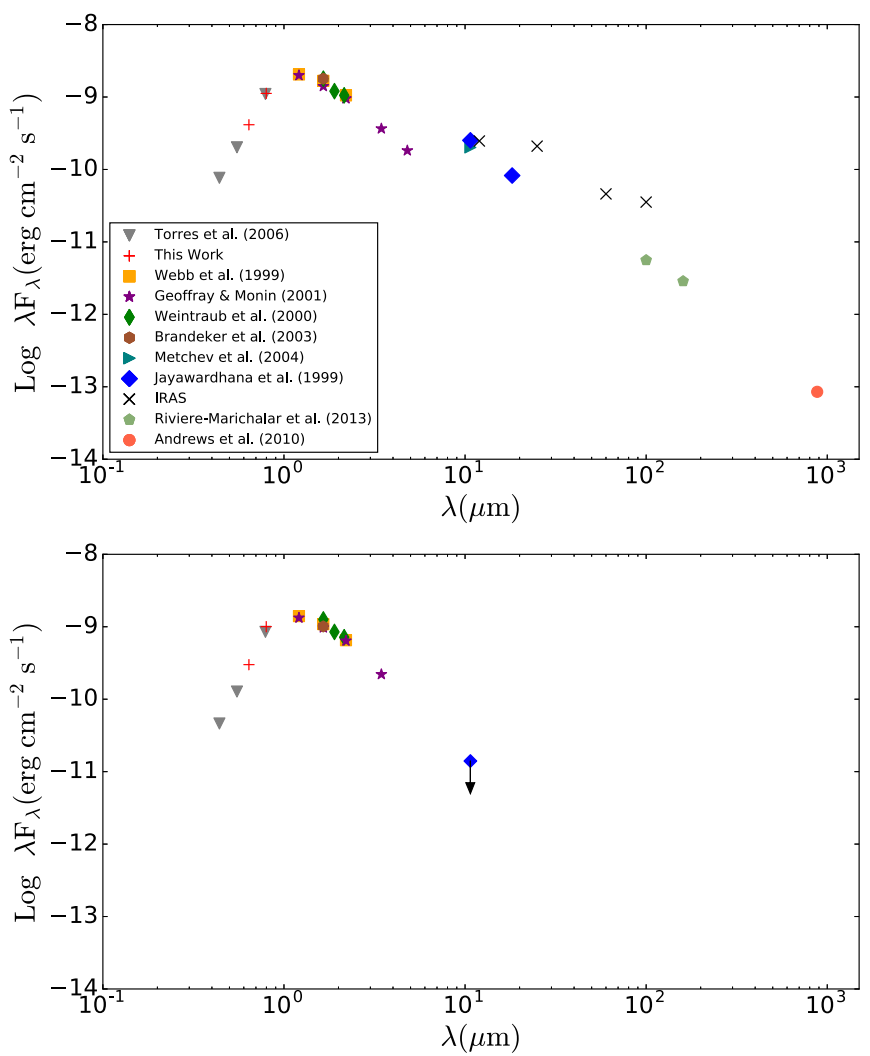

Figure 7. SEDs based primarily on data from the literature; sources and references for the plotted points are given in boldface in Table 13. Top: TWA 3A; bottom: TWA 3B.

unambiguous detection of active gas accretion onto at least one star in the TWA 3Aa-Ab pair, although they calculate a relatively low mass accretion rate of $5 \times 10^{-11} M_{\odot} \mathrm{yr}^{-1}$.

We fit our SEDs with the solar-metallicity $(\mathrm{Fe} / \mathrm{H}$ within \pm 0.05 ) spectral templates from Mann et al. (2015) using both our broad- and narrowband photometry (Table 13) taken at $\lambda<3.0 \mu \mathrm{m}$. For the $I_{C}$ and $R_{C}$ filters, we averaged the values for the angularly unresolved TWA 3 system given in Table 13 and used the A/B flux ratios in Table 4 to calculate the individual $\mathrm{A}$ and $\mathrm{B}$ component magnitudes and corresponding fluxes. Fixing $A_{V}$ to a value of 0.01 mag (McJunkin et al. 2014) and scaling the flux of the spectral templates to match the observed fluxes resulted in a best fit for TWA 3A to the M4.1 template (PM I19321-1119) and for TWA 3B to the M3.5 template (PM I09553-2715), consistent with our optimal 


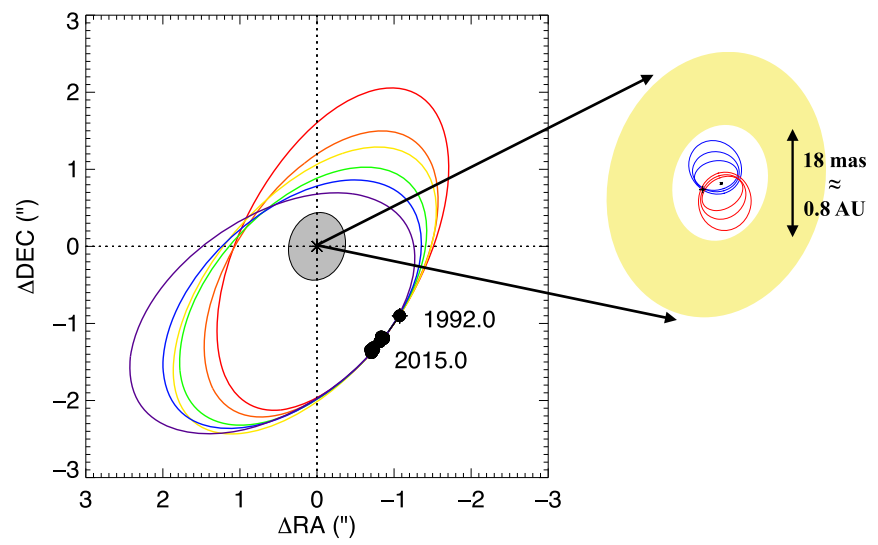

Figure 8. Binary component orbits and disk orientation for the TWA 3 system. The gray ellipse shows the orientation and outer radius of 20 au for the $880 \mu \mathrm{m}$ circumbinary dust disk around TWA 3A, deconvolved from the beam size (Andrews et al. 2010). The inset shows a blow-up of the inner cavity of tenuous gas with an inside radius of 0.4 au and an outer radius of 1.0 au (Andrews et al. 2010). To convert the physical dimensions used by Andrews et al. to an angular scale for comparison with our orbit families, we used the distance cited by Andrews et al., 45 pc.

cross-correlation results. At a distance of $34 \mathrm{pc}$, summing the flux under the model SED (using the Rayleigh-Jeans tail of a blackbody curve to approximate the flux at wavelengths longer than $3 \mu \mathrm{m}$ ) yields luminosities of $0.081 \pm 0.003 \pm 0.022 L_{\odot}$ and $0.055 \pm 0.002 \pm 0.015 L_{\odot}$ for the A and B components, respectively. The first uncertainty is computed from the SED fit, while the second is from propagating the \pm 4 pc uncertainty in the distance.

\subsection{Disk-Orbit Alignment}

According to the model of Andrews et al. (2010), the TWA $3 \mathrm{~A}$ circumbinary disk inclination is $36^{\circ}$ and the position angle is $169^{\circ}$ east of north. In Figure 8 we show a schematic of this model disk with the family of probable orbital fits to the arc of observed positions (Figure 5). In order to calculate the relative inclination between the disk and the outer orbit of TWA 3B, we followed the approach of Fekel (1981) for calculating the relative orbital inclination $\phi$ in a hierarchical triple by substituting the disk's position angle and inclination for the inner orbit and a typical orbit from those shown in Figure 8 for the outer orbit $(P=671.73 \mathrm{yr}, T=2451805.0, e=0.3976$, $a=2$ " 100 or 71.40 au at $d=34 \mathrm{pc}, i=124^{\circ} .91, \Omega=133^{\circ} .24$, $\left.\omega=133.43, M_{\text {total }}=0.807 M_{\odot}\right)$. The disk's position angle is that of the line of nodes and is not necessarily the position angle of the ascending node $\Omega_{\mathrm{disk}}$, required by the Fekel formula, because it is not possible to distinguish the ascending node from the descending node without RVs for the disk, which have not been measured. Because of this $180^{\circ}$ ambiguity in $\Omega_{\text {disk }}$, the calculation of $\phi$ yields two results; for the plus sign in Fekel's equation we obtain $\phi=86^{\circ}$ and for the minus sign we find $\phi=32^{\circ}$. This analysis suggests a significant discrepancy between the disk inclination and wide orbit inclination; it is even possible that these are close to perpendicular.

We applied the same experiment to the disk with respect to the inner binary. Restricting the orbital parallax to be within $34 \pm 4$ pc, we determined the median inclinations and values for $\Omega$ along with their associated $1 \sigma$ uncertainties for the two families of orbits shown in Figure 4: $i=136_{-18}^{\circ}$, $\Omega=103^{\circ} .4 \pm 6.4$ and $i=43_{-12 \circ}^{\circ}, \quad \Omega=112.3 \pm 6.6$. For the former inner orbit family, the plus sign in Fekel's equation yielded $\phi=139^{\circ}$ and the minus sign $\phi=114^{\circ}$. Even with uncertainties on the order of $\pm 20^{\circ}$, this implies a highly misaligned and retrograde orbit. For the latter inner orbit family, the plus sign in Fekel's equation yielded $\phi=36^{\circ}$ and the minus sign $\phi=68^{\circ}$, implying that a significant misalignment is also possible for these values. We can also use the range of values for the semimajor axes in the two families of orbits consistent with the very long baseline interferometry (VLBI) measurement shown in Figure 4, $a=6.5-4.7$ mas, together with the value for $a \sin i$ given in Table 10 for the combined IR and visible light spectroscopic binary solution, $27.34 R_{\odot}$, assuming a distance of 34 pc. For $a=6.5$ mas we obtain an inclination of $35^{\circ}$ and for $a=4.7$ mas an inclination of $53^{\circ}$, a similar range to that described in the latter calculation above.

In Figure 8 we show the typical inner orbits as in Figure 4 with a blow-up of the inner circumbinary disk. It appears likely that neither the inner spectroscopic binary orbit nor the outer visual binary orbit is coplanar with the disk. In contrast, Czekala et al. (2016) found that the $\sim 16$-day period DQ Tau spectroscopic binary is aligned with its circumbinary disk to within $3^{\circ}$ at the $3 \sigma$ level. This discrepancy in the TWA 3 system may be related to the presence of a torque on the disk originating in the action of the tertiary.

\subsection{Origin of the TWA 3 System}

The TWA 3 system presents a counterintuitive example of a young hierarchical triple with no evidence for accretion disk material around the single star in the wide, several-hundredyear orbit, but an actively accreting circumbinary disk surrounding the two stars in a $\sim 35$-day spectroscopic binary orbit. Given its disk characteristics, TWA 3A falls into the rare class of double-lined, CTTS spectroscopic binaries along with other examples such as DQ Tau (Czekala et al. 2016), V4046 Sgr (Rosenfeld et al. 2012), and UZ Tau E (Prato et al. 2002b; Martín et al. 2005). Uniquely, however, TWA 3 is the lowestmass system in this group, and one of the oldest. Furthermore, not only is the TWA 3A spectroscopic binary surrounded by an actively accreting disk, but it is also accompanied by a tertiary low-mass stellar companion that lacks any evidence of disk material. If the three stellar components in this system formed concurrently, we are left with the question of why only the close binary hosts a disk. One obvious advantage is the higher mass of the double star in terms of gravitational potential, but this is offset by the disadvantage of the dynamical impact on disk material by the orbital action of the close binary.

Considering the available evidence to the effect that the A-B pair appears bound (Section 4.3), it is attractive to ascribe the mismatched disk properties of these wide components to the result of a dynamical capture event. Clarke \& Pringle (1991), however, show that capture rates in the known star-forming regions, even of a star by a massive disk, are too low to provide an important binary formation mechanism. The disk surrounding TWA 3A has below-average dust mass at $7 \times 10^{-6} M_{\odot}$ (Andrews et al. 2010), although it was likely more massive when the system was newly formed. Very wide pairs (separation $>1000 \mathrm{au}$ ) can also form through capture in the late stages of cluster dispersal (e.g., Kouwenhoven et al. 2010); the probable family of orbits shown in Figure 5, however, possesses semimajor axes between 70 and $80 \mathrm{au}$, too tight for the soft binaries formed as per Kouwenhoven et al. during 
cluster dissolution. Alternatively, the TWA 3 system could have been a loose aggregate of stars that was subsequently reconfigured as a tight binary and a relatively wide tertiary (e.g., Reipurth \& Mikkola 2012). In this case, the closer binary's interaction with the disk material would tighten the orbit, and the TWA 3B tertiary would be isolated outside of the binary+disk system. However, the $N$-body simulations of Reipurth \& Mikkola treat the evolution of compact triple systems that evolve to a configuration with a tight pair and an extremely distant (i.e., hundreds of au) tertiary, a scale far greater than that we observe in the TWA 3 triple.

Kaplan et al. (2012) examined the probability of a purely gravitational interaction between a close brown dwarf binary and a solar-type star. Although TWA 3 is composed simply of three low-mass stars, we explored the results of their work in search of clues to possible formation mechanisms. The likelihood of capture in the brown dwarf pair-solar-type star model is $\sim 0.1 \%$ and thus unlikely. However, given a low-mass tertiary, the possibility of capture could be higher, although it seems unlikely.

\section{Discussion}

In spite of the nominal age of $10 \mathrm{Myr}$ for the TW Hya association (e.g., Webb et al. 1999), consistent with our results (Figure 6), ongoing gas accretion surprisingly points to an actively accreting circumbinary disk around TWA 3A. The eccentric binary is expected to perturb such a disk, clearing out a hole in the center; Aguilar et al. (2008) find that the higher the eccentricity of a system, the larger the inner radius of the circumbinary disk. Figure 8 shows possible visual orbits of the spectroscopic binary compared to the size of the tenuous inner disk, 0.4-1.0 au, as modeled by Andrews et al. (2010). Given the large eccentricity of the spectroscopic binary of $\sim 0.63$, this tenuous gas disk cannot be stable under the criteria of Artymowicz \& Lubow (1994) and likely represents the streaming gas from the disk outside of 1 au to the accretion point with the stars, just inside of $0.4 \mathrm{au}$. Yang et al. (2012) measured the far-UV emission in TWA $3 \mathrm{~A}$ and $3 \mathrm{~B}$ and detected $\mathrm{H}_{2}$ emission lines and a continuum excess in the $\mathrm{A}$ component but not in $\mathrm{B}$. Although they point out that the low Ca II/C IV luminosity ratio of TWA $3 \mathrm{~A}$ could result from purely chromospheric activity, the strong $\mathrm{H} \alpha$ emission, $\mathrm{H}_{2}$ emission, and Balmer continuum emission all point to active accretion from the TWA $3 \mathrm{~A}$ circumbinary disk onto one or both of the central stars. Herczeg et al. (2009) report the presence of [O I] emission around TWA $3 \mathrm{~A}$ as well, indicative of outflow.

The lack of near-IR excess emission reflects the destruction of the inner dust disk by the action of the spectroscopic binary orbit, leaving only the tenuous gas (Andrews et al. 2010) detected by Yang et al. (2012) as hot $\mathrm{H}_{2}$ and by Webb et al. (1999), Muzerolle et al. (2000), and Herczeg et al. (2009) in the $\mathrm{H} \alpha$ emission line. Interestingly, the crystalline silicate emission detected by Uchida et al. (2004) points to significant thermal processing in the dust outside the central cavity, delineated by the modeling of Andrews et al. (2010) at $\sim 1$ au. Herczeg \& Hillenbrand (2014) determine a relatively young age of 3-4 Myr for the TWA 3 system, in contrast to the canonical $\sim 10$ Myr for TW Hya association stars and consistent with our results.

Although a number of very young systems such as UZ Tau E (Prato et al. 2002b), DQ Tau (Basri et al. 1997), and AS 205B
(Eisner et al. 2005) possess actively accreting, circumbinary disks, these systems have ages of just 1 to a few Myr. Other components in these systems, such as UZ Tau W and AS 205A, also boast active, optically thick disks. What challenges our understanding of disk evolution in the much older TWA 3 system is the unexpected presence of an active disk around the close binary rather than around the single visual companion. It is possible, given the relatively short period and high eccentricity, as well as the presence of TWA $3 \mathrm{~B}$ at only $\sim 70$ $\mathrm{au}$, that the eccentric spectroscopic orbit of TWA 3A may have been impacted by the Kozai pumping induced by TWA 3B. Another 10 Myr old system, TWA 4 (HD 98800), is similarly puzzling: a quadruple system in which the visual companions are themselves spectroscopic binaries (Torres et al. 1995), with evidence for a circumbinary disk only around the visual secondary component, TWA 4B (e.g., Prato et al. 2001). As Torres et al. showed, the TWA 4B pair has a high eccentricity, $\sim 0.8$, but the diskless single-lined binary, TWA 4A, also has a relatively high eccentricity of $\sim 0.5$.

It has been speculated that the existence of a companion will prolong the depletion of the circumstellar disk and slow the rotation of the stars (Armitage \& Clarke 1996). If this is correct, then it might explain why there is still a disk present in the system but not why it is around the spectroscopic binary component of the system rather than the single-star component. Bouvier et al. (1997) and White \& Hillenbrand (2005) suggested that disk truncation and tidal interactions inhibit accretion flow, which would thus increase the lifetime of this disk as well. This theory is contrary to evidence suggesting that the circumbinary disk around TWA 3A is still accreting, however. Lubow \& Artymowicz (1997) showed that if the internal gas pressure in the circumbinary disk is large enough to overcome the energy barrier of the resonance, inward gas streams could flow across the gap onto the central stars. This could then explain why the system is still accreting, but it cannot explain the lifetime of the disk.

If indeed TWA 3A and 3B are bound and both formed with disks, we speculate that the same sequence of tidal interactions invoked by Prato et al. (2001) may have disrupted the shorttimescale dispersal of the TWA 3B disk. Prato et al. studied the properties of the quadruple TWA 4 and hypothesized that both of the tight, spectroscopic pairs in that system may have originally hosted circumbinary disks, but that the difference in relative disk inclinations may have ripped the circumbinary disk from TWA 4A early in the formation history of the system. Given the apparent lack of alignment, however, between either of the stellar binary orbits and the disk plane, justification for the survival of the lone circumbinary disk is not obvious. At best we might evoke a particular set of parameters that ensure the stability and contribute to the longevity even of the remaining disk.

Future observations that could ultimately help define the system are straightforward for the inner orbit and circumbinary disk: even a small number of additional VLBI visibilities will facilitate measurement of the visual orbit and hence individual masses for the 35-day period binary. ALMA observations of the disk will refine its inclination and $\Omega$ values and at high spatial resolution may reveal structure and detail both within the disk and of the outer radius. Although numerous historical plates of the TWA 3 system have been digitized, the extremely poor image resolution makes analysis of the relative positions of the 1 1"55 binary impossible. To realize a more definitive 
TWA 3A-B orbit, it will likely be necessary to wait another 20 or $40 \mathrm{yr}$.

\section{Summary}

We report a $\sim 35$-day period spectroscopic companion to the visual primary star in the TWA 3 system. We find that the primary component (Aa) of the spectroscopic binary has an M4 spectral type with a $v \sin i$ of $\sim 7 \mathrm{~km} \mathrm{~s}^{-1}$ and the secondary component $(\mathrm{Ab})$ has an M4.5 spectral type with a $v \sin i$ of $\sim 5 \mathrm{~km} \mathrm{~s}^{-1}$. As expected for these spectral types, the two stars in the spectroscopic binary have a relatively high mass ratio, $0.841 \pm 0.014$. TWA $3 \mathrm{~B}$, the visual companion at 1.155 , has an M3.5 spectral type with a $v \sin i$ of $\sim 12 \mathrm{~km} \mathrm{~s}^{-1}$ and is apparently single. Values of $v \sin i$ given here are taken from the optical data sets (Section 3.2). Based on all available data, from our own spectroscopic solution for TWA 3A as well as multiple sources from the literature, we have determined that the one disk present in the TWA 3 system orbits only the spectroscopic pair and is not aligned with either the shortperiod inner or long-period outer orbit. We speculate that the longevity and apparent stability of this disk are related to the unusual dynamics in this complex system, but we are at a loss to explain the lack of any circumstellar material orbiting TWA $3 \mathrm{~B}$, if indeed this component formed with a disk. All indications, relative motion, age, and formation hypotheses, are consistent with the TWA 3A and 3B components being stably bound. TWA 3 shares the multiple-star complexity and disk longevity with several other prominent systems in the TW Hya region, such as TWA 4, TWA 1, and TWA 5. We conjecture that relatively unusual initial conditions in this small association contributed not only to the high multiplicity fraction of the members but also possibly to the long-lived and unusual disk configurations. These properties may also be related at least in part to an observational bias in that the TW Hya association is located at $<50 \mathrm{pc}$, allowing detailed study not only of the disk properties but also of both visual and spectroscopic binaries.

We thank numerous observatory TOs, OAs, and SAs for their exceptional observing support over the many years of data collection for this project. L.P. thanks Otto Franz, Cathie Clarke, and Steve Lubow, and Peter Bodenheimer among others, for helpful discussions that improved this manuscript. We are grateful to P. Berlind, M. Calkins, G. Esquerdo, D. Latham, and R. Stefanik for help in obtaining the CfA observations of TWA 3, and to R. J. Davis for maintaining the CfA echelle database over the years. L.P. thanks A.B. for inspiring discussions during the completion of this work. We are extremely grateful to the rapid response of the referee and to the referee's comments and suggestions, which have improved this manuscript. L.P., K.K., I.A., L.H.W., and D.R. R. were supported in part by NSF grants AST-1009136 and AST-1313399 (to L.P.). Initial work on this project by K.K. and I.A. was also supported by a NASA Space Grant, through Northern Arizona University. G.T. acknowledges partial support for this work from NSF grant AST-1509375. G.H.S. acknowledges support from NSF grant AST-1411654. K.M. M.'s and L.M.C.'s work was supported by the NASA Exoplanets Research Program (XRP) by cooperative agreement NNX16AD44G. These results made use of the Discovery Channel Telescope at Lowell Observatory. Lowell is a private, nonprofit institution dedicated to astrophysical research and public appreciation of astronomy and operates the DCT in partnership with Boston University, the University of Maryland, the University of Toledo, Northern Arizona University, and Yale University. The Large Monolithic Imager was built by Lowell Observatory using funds provided by the National Science Foundation (AST-1005313). We thank Sean Andrews for providing us with the $880 \mu \mathrm{m}$ dust continuum contours from Andrews et al. (2010) and Bo Reipurth for locating the exact date of his first measurement of the TWA $3 \mathrm{~A}-\mathrm{B}$ pair shown in Reipurth \& Zinnecker (1993). We are grateful to T. Boyajian for a useful discussion on the best templates to use in the SED fits and to A. Mann for providing the spectral templates. This research has made use of the Washington Double Star Catalog maintained at the U.S. Naval Observatory and the SIMBAD reference database, the NASA Astrophysics Data System, and the data products from the Two Micron All Sky Survey, which is a joint project of the University of Massachusetts and the Infrared Processing and Analysis Center/California Institute of Technology, funded by the National Aeronautics and Space Administration and the National Science Foundation. Data presented herein were obtained at the W. M. Keck Observatory from telescope time allocated to the National Aeronautics and Space Administration through the agency's scientific partnership with the California Institute of Technology and the University of California. The Observatory was made possible by the generous financial support of the W. M. Keck Foundation. We recognize and acknowledge the significant cultural role that the summit of Maunakea plays within the indigenous Hawaiian community and are grateful for the opportunity to conduct observations from this special mountain.

\section{References}

Aguilar, L. A., Pichardo, B. S., \& Sparke, L. S. 2008, RMxAC, 34, 91 Andrews, S. M., Czekala, I., Wilner, D. J., et al. 2010, ApJ, 710, 462 Anthonioz, F., Ménard, F., Pinte, C., et al. 2015, A\&A, 574, A41 Armitage, P. J., \& Clarke, C. J. 1996, MNRAS, 280, 458 Artymowicz, P., \& Lubow, S. H. 1994, ApJ, 421, 651 Baraffe, I., Homeier, D., Allard, F., \& Chabrier, G. 2015, A\&A, 577, A43 Basri, G., Johns-Krull, C. M., \& Mathieu, R. D. 1997, AJ, 114, 781 Beichman, C. A., Neugebauer, G., Habing, H. J., Clegg, P. E., \& Chester, T. J. 1988, Infrared Astronomical Satellite (IRAS) Catalogs and Atlases, Vol. 1: Explanatory Supplement, 1

Bender, C., Simon, M., Prato, L., Mazeh, T., \& Zucker, S. 2005, AJ, 129, 402 Bender, C. F., \& Simon, M. 2008, ApJ, 689, 416

Bouvier, J., Alencar, S. H. P., Harries, T. J., Johns-Krull, C. M., \& Romanova, M. M. 2007, in Protostars and Planets V, ed. B. Reipurth, D. Jewitt, \& K. Keil (Tucson, AZ: Univ. of Arizona Press), 479 Bouvier, J., Rigaut, F., \& Nadeau, D. 1997, A\&A, 323, 139

Brandeker, A., Jayawardhana, R., \& Najita, J. 2003, AJ, 126, 2009

Browning, M. K., Basri, G., Marcy, G. W., West, A. A., \& Zhang, J. 2010, AJ, 139,504

Clarke, C. J., \& Pringle, J. E. 1991, MNRAS, 249, 584

Correia, S., Zinnecker, H., Ratzka, T., \& Sterzik, M. F. 2006, A\&A, 459, 909 Cutri, R. M., Skrutskie, M. F., van Dyk, S., et al. 2003, yCat, 2246, 0

Cutri, R. M. 2012, yCat, 2311, 0

Czekala, I., Andrews, S. M., Torres, G., et al. 2016, ApJ, 818, 156

de la Reza, R., Torres, C. A. O., Quast, G., Castilho, B. V., \& Vieira, G. L. 1989, ApJL, 343, L61

Dotter, A., Chaboyer, B., Jevremović, D., et al. 2008, ApJS, 178, 89

Doyle, L. R., Carter, J. A., Fabrycky, D. C., et al. 2011, Sci, 333, 1602

Duchêne, G., \& Kraus, A. 2013, ARA\&A, 51, 269

Eisner, J. A., Hillenbrand, L. A., White, R. J., Akeson, R. L., \& Sargent, A. I. 2005, ApJ, 623, 952

Fekel, F. C., Jr. 1981, ApJ, 246, 879

Gagné, J., Faherty, J. K., Mamajek, E. E., et al. 2017, ApJS, 228, 18

Geoffray, H., \& Monin, J. L. 2001, A\&A, 369, A239

Goldin, A., \& Makarov, V. V. 2007, ApJS, 173, 137 
Gregorio-Hetem, J., Lepine, J. R. D., Quast, G. R., Torres, C. A. O., \& de La Reza, R. 1992, AJ, 103, 549

Guilloteau, S., Simon, M., Pietu, V., et al. 2014, A\&A, 567, A117

Haisch, K. E., Jr., Lada, E. A., \& Lada, C. J. 2001, ApJL, 553, L153

Herczeg, G. J., Cruz, K. L., \& Hillenbrand, L. A. 2009, ApJ, 696, 1589

Herczeg, G. J., \& Hillenbrand, L. A. 2014, ApJ, 786, 97

Hernández, J., Hartmann, L., Calvet, N., et al. 2008, ApJ, 686, 1195

Huenemoerder, D. P., Kastner, J. H., Testa, P., Schulz, N. S., \& Weintraub, D. A. 2007, ApJ, 671, 592

Janson, M., Bergfors, C., Brandner, W., et al. 2014, ApJS, 214, 17

Jayawardhana, R., Hartmann, L., Fazio, G., et al. 1999, ApJL, 520, L41

Kaplan, M., Stamatellos, D., \& Whitworth, A. P. 2012, Ap\&SS, 341, 395

Kellogg, K., Metchev, S., Gagné, J., \& Faherty, J. 2016, ApJL, 821, L15

Kim, S., Prato, L., \& McLean, I. 2015, REDSPEC: NIRSPEC data reduction, Astrophysics Source Code Library, ascl:1507.017

Koerner, D. W., Jensen, E. L. N., Cruz, K. L., Guild, T. B., \& Gultekin, K. 2000, ApJL, 533, L37

Konopacky, Q. M., Ghez, A. M., Duchêne, G., McCabe, C., \& Macintosh, B. A. 2007, AJ, 133, 2008

Kostov, V. B., McCullough, P. R., Carter, J. A., et al. 2014, ApJ, 784, 14

Kouwenhoven, M. B. N., Goodwin, S. P., Parker, R. J., et al. 2010, MNRAS, 404, 1835

Latham, D. W. 1992, in ASP Conf. Ser. 32, IAU Coll. 135, Complementary Approaches to Double and Multiple Star Research, ed. H. A. McAlister \& W. I. Hartkopf (San Francisco, CA: ASP), 110

Leggett, S. K., Allard, F., Geballe, T. R., Hauschildt, P. H., \& Schweitzer, A. 2001, ApJ, 548, 908

Le Bouquin, J. B., Monin, J. L., Berger, J. P., et al. 2014, A\&A, 561, A101

Lubow, S. H., \& Artymowicz, P. 1997, in ASP Conf Ser. 121, Accretion Phenomena and Related Outflows, ed. D. T. Wickramasinghe, G. V. Bicknell, \& L. Ferrario (San Francisco, CA: ASP), 505

Luhman, K. L., Allen, P. R., Espaillat, C., Hartmann, L., \& Calvet, N. 2010, ApJS, 186, 111

Luhman, K. L., Briceño, C., Stauffer, J. R., et al. 2003, ApJ, 590, 348

Malo, L., Artigau, É., Doyon, R., et al. 2014, ApJ, 788, 81

Mamajek, E. E. 2005, ApJ, 634, 1385

Mann, A. W., Feiden, G. A., Gaidos, E., Boyajian, T., \& von Braun, K. 2015, ApJ, 804, 64

Martín, E. L. 1998, AJ, 115, 351

Martín, E. L., Magazzù, A., Delfosse, X., \& Mathieu, R. D. 2005, A\&A, 429, 939

Mason, B. D., Wycoff, G. L., Hartkopf, W. I., Douglass, G. G., \& Worley, C. E. 2001, AJ, 122, 3466

Mathieu, R. D., Adams, F. C., \& Latham, D. W. 1991, AJ, 101, 2184

Mazeh, T., Simon, M., Prato, L., Markus, B., \& Zucker, S. 2003, ApJ, 599, 1344

McJunkin, M., France, K., Schneider, P. C., et al. 2014, ApJ, 780, 150

Metchev, S. A., Hillenbrand, L. A., \& Meyer, M. R. 2004, ApJ, 600, 435

Morzinski, K. M., Males, J. R., Skemer, A. J., et al. 2015, ApJ, 815, 108
Munari, U., Henden, A., Frigo, A., et al. 2014, AJ, 148, 81

Murdoch, K. A., Hearnshaw, J. B., \& Clark, M. 1993, ApJ, 413, 349

Muzerolle, J., Calvet, N., Briceño, C., Hartmann, L., \& Hillenbrand, L. 2000, ApJL, 535, L47

Pickles, A. J. 1998, PASP, 110, 863

Prato, L. 2007, ApJ, 657, 338

Prato, L., Ghez, A. M., Piata, R. K., et al. 2001, ApJ, 549, 590

Prato, L., Simon, M., Mazeh, T., et al. 2002a, ApJ, 569, 863

Prato, L., Simon, M., Mazeh, T., Zucker, S., \& McLean, I. S. 2002b, ApJL, 579, L99

Press, W. H., Teukolsky, S. A., Vetterling, W. T., \& Flannery, B. P. 1992, Numerical Recipes (2nd ed.; Cambridge: Cambridge Univ. Press), 650

Reiners, A., Joshi, N., \& Goldman, B. 2012, AJ, 143, 93

Reipurth, B., \& Mikkola, S. 2012, Natur, 492, 221

Reipurth, B., \& Zinnecker, H. 1993, A\&A, 278, 81

Riviere-Marichalar, P., Pinte, C., Barrado, D., et al. 2013, A\&A, 555, A67

Rosenfeld, K. A., Andrews, S. M., Wilner, D. J., \& Stempels, H. C. 2012, ApJ, 759, 119

Rosero, V., Prato, L., Wasserman, L. H., \& Rodgers, B. 2011, AJ, 141, 13

Rousselot, P., Lidman, C., Cuby, J.-G., Moreels, G., \& Monnet, G. 2000, A\&A, 354, 1134

Schaefer, G. H., Prato, L., Simon, M., \& Patience, J. 2014, AJ, 147, 157

Schaefer, G. H., Simon, M., Prato, L., \& Barman, T. 2008, AJ, 135, 1659

Simon, M., Dutrey, A., \& Guilloteau, S. 2000, ApJ, 545, 1034

Simon, M., Schaefer, G. H., Prato, L., et al. 2013, ApJ, 773, 28

Steffen, A. T., Mathieu, R. D., Lattanzi, M. G., et al. 2001, AJ, 122, 997

Tokovinin, A., Mason, B. D., Hartkopf, W. I., Mendez, R. A., \& Horch, E. P. 2015, AJ, 150, 50

Torres, C. A. O., da Silva, L., Quast, G. R., de La Reza, R., \& Jilinski, E. 2000, AJ, 120, 1410

Torres, C. A. O., Quast, G. R., da Silva, L., et al. 2006, A\&A, 460, 965

Torres, G., Latham, D. W., \& Stefanik, R. P. 2007, ApJ, 662, 602

Torres, G., Mader, J. A., Marschall, L. A., Neuhäuser, R., \& Duffy, A. S. 2003 AJ, 125, 3237

Torres, G., Stefanik, R. P., Latham, D. W., \& Mazeh, T. 1995, ApJ, 452, 870

Uchida, K. I., Calvet, N., Hartmann, L., et al. 2004, ApJS, 154, 439

Webb, R. A., Zuckerman, B., Platais, I., et al. 1999, ApJL, 512, L63

Weinberger, A. J., Becklin, E. E., Schneider, G., et al. 2002, ApJ, 566, 409

Weintraub, D. A., Saumon, D., Kastner, J. H., \& Forveille, T. 2000, ApJ, 530,867

Welsh, W. F., Orosz, J. A., Short, D. R., et al. 2015, ApJ, 809, 26

White, R. J., Hillenbrand, L., Metchev, S., \& Patience, J. 2002, BAAS, 34, 1134

White, R. J., \& Hillenbrand, L. A. 2005, ApJL, 621, L65

Wilson, O. C. 1941, ApJ, 93, 29

Yang, H., Herczeg, G. J., Linsky, J. L., et al. 2012, ApJ, 744, 121

Zucker, S., \& Mazeh, T. 1994, ApJ, 420, 806

Zucker, S., Torres, G., \& Mazeh, T. 1995, ApJ, 452, 863

Zuckerman, B. 2001, ARA\&A, 39, 549 